UCRL-JC-124651

PREPRINT

\title{
Observation of Multiple Mechanisms for Stimulating Ion Waves in Ignition Scale Plasmas
}

R. K. Kirkwood, B. J. MacGowan, D. S. Montgomery, B. B. Afeyan, W. L. Kruer, D. M. Pennington, S. C. Wilks, J. D. Moody, C. A. Back, K. G. Estabrook, S. H. Glenzer, M. A. Blain, C. Rousseaux, E. A. Williams, R. L. Berger, B. F. Lasinski

This paper was prepared for submittal to the

1996 Division of Plasma Physics of the American Physical Society

Denver, $\mathrm{CO}$

November 11-15, 1996

November 1, 1996

This is a preprint of a paper intended for publication in a journal or proceedings.

Since changes may be made before publication, this preprint is made available with the understanding that it will not be cited or reproduced without the permission of the author. 


\section{DISCLAIMER}

This document was prepared as an account of work sponsored by an agency of the United States Government. Neither the United States Government nor the University of California nor any of their employees, makes any warranty, express or implied, or assumes any legal liability or responsibility for the accuracy, completeness, or usefulness of any information, apparatus, product, or process

disclosed, or represents that its use would not infringe privately owned rights. Reference herein to any specific commercial product, process, or service by trade name, trademark, manufacturer, or otherwise, does not necessarily constitute or imply its endorsement, recommendation, or favoring by the United States Government or the University of California. The views and opinions of authors expressed herein do not necessarily state or reflect those of the United States Government or the University of California, and shall not be used for advertising or product endorsement purposes. 
Invited Paper for 1996 meeting of

the Division of Plasma Physics of the American Physical Society

\section{Observation of Multiple Mechanisms For Stimulating Ion Waves in Ignition Scale Plasmas}

R. K. Kirkwood,B. J. MacGowan, D. S. Montgomery, B. B. Afeyan, W. L. Kruer, D. M. Pennington, S. C. Wilks, J. D. Moody, K. Wharton, C. A. Back, K. G. Estabrook, S. H. Glenzer, M. A. Blaint, R. L. Berger, D. E. Hinkel, B. F. Lasinski, E. A. Williams, D. Munro, B. H. Wildett, and C. Rousseauxt Lawrence Livermore National Laboratory USA †Centre D'Etudes de Limeil-Valenton, France t+Los Alamos National Laboratory USA

\section{Abstract}

The laser and plasma conditions expected in ignition experiments using indirect drive inertial confinement have been studied experimentally. It has been found that there are at least three ways in which ion waves can be stimulated in these plasmas and have significant effect on the energy balance and distribution in the target. First ion waves can be stimulated by a single laser beam by the process of Stimulated Brillouin Scattering (SBS) in which an ion acoustic and a scattered electromagnetic wave grow from noise.

Second, in a plasma where more than one beam intersect, ion waves can be excited at the 'beat' frequency and wave number of the intersecting beams, causing the side scatter instability to be seeded, and substantial energy to be transferred between the beams [R. K. Kirkwood et. al. Phys. Rev. Lett. 76, 2065 (1996)]. And third, ion waves may be stimulated by the decay of electron plasma waves produced by Stimulated Raman Scattering (SRS), thereby inhibiting the SRS process [R. K. Kirkwood et. al. Phys. Rev. Lett. 77, 2706 (1996)]. 


\section{Introduction}

In order to ignite a fusion reaction with intense laser beams it is necessary for the beams propagate to the surface of the solid density material without significant loss or angular redistribution of energy. In the region near the surface of the solid, a plasma is formed. The stimulation of both ion acoustic waves (SBS) and Langmuir waves (SRS) in this plasma can scatter the laser beams at a variety of angles. To achieve ignition by the 'indirectdrive' approach in which the laser beams impinge on the interior surface of a gold cavity or hohlraum which surrounds the fusion fuel, the interior of the hohlraum must also be filled with a low atomic number $(\mathrm{Z})$ gas that retards the motion of the gold interface [1]. As a result the beams must transit a region filled with a low density, low $\mathrm{Z}$ plasma created by the gas as well as a region filled with a high $\mathrm{Z}$ Au plasma created when the wall ablates. Because of laser absorbtion and thermal transport the plasma in this region has typical electron temperatures of 4 to $5 \mathrm{keV}$ and density and velocity scale lengths in the range of $100 \mu \mathrm{m}$ to $2 \mathrm{~mm}$ with densities that are $\sim 0.1$ the critical density for the incident light. Recently, we have developed the capability to simulate these conditions [2] using the Nova laser facility where we have determined the fraction of the energy that is back scattered by the direct stimulation of ion waves and Langmuir waves in the plasmas expected in ignition experiments [3].

In this paper we describe multiple mechanisms by which ion waves can be stimulated in ignition experiments and how they affect energy deposition on the target. In addition to studies of SBS produced by a single laser beam, experiments have been performed to characterize the scattering by ion waves produced by two intersecting laser beams that have a frequency separation matched to the ion wave frequency [4], as well as the effect of the 
secondary stimulation of ion waves by the daughter Langmuir waves produced by the SRS process [5]. These experiments each address a three wave decay mechanism that can produce ion waves in an ignition experiment and quantify the effect of each on the energy delivered to the target. The three different mechanisms that have been identified as important in this application are shown schematically in Figure 1. The first mechanism, SBS, involves an ion wave that grows from thermal noise, driven by the incident and scattered electromagnetic waves. The electromagnetic wave scattered by the thermal ion wave beats with the incident electromagnetic wave and forms a ponderomotive force 'grating' that has the same wave vector as the scattering ion wave. When this grating also has the same frequency as the ion wave it causes the wave to grow in space (and time) until it is saturated by some mechanism such as non-linear effects or convection of energy out of the volume by one or both of the stimulated waves. Because the growth rate is often largest when the scattered wave is propagating opposite to the incident beam (back scattering) the result of the instability is usually to reduce the energy deposited on the target. SBS has been described extensively in earlier presentations and papers [3,6,7] and the references therein, and will only be considered briefly here. The second mechanism shown in Figure 1 is the interaction of two crossing laser beams that have a difference frequency that is equal to that of the resonant ion wave [4]. The instability process driven by the two beams is essentially the stimulated Brillouin side scatter (SBSS) process described above, but which grows from a seed electromagnetic wave provided by the second beam, rather than a seed ion wave produced by thermal noise $[8,9]$. This instability scatters energy from one beam into another, causing the redistribution of energy among the beams rather than the back scattering of energy produced by single beam SBS. Because the seed 
wave is a second laser beam rather than a small amplitude thermal ion wave, substantial energy can be transferred even when the side scattering gain is low. The third mechanism shown in Figure 1 is the secondary decay of the SRS generated Langmuir wave into an ion wave and a third wave. To drive the instability the SRS mechanism must first grow a large amplitude Langmuir wave by a mechanism that is similar to the SBS mechanism described above except that a Langmuir wave scatters the incident beam rather than an ion wave. In order for the Langmuir wave to undergo the secondary decay it must achieve an amplitude above the threshold for the decay process in spite of any other saturation mechanisms that may be operating. Once the threshold is reached the Langmuir wave then decays itself through one of two resonant three wave processes which create the following daughter waves: an ion wave and either an electromagnetic wave, if the decay is due to the electromagnetic decay instability (EDI), or a second Langmuir wave, if the decay is due to the Langmuir decay instability (LDI). These processes drain energy from the SRS driven Langmuir wave and impede the growth of the primary SRS process, thereby reducing the back scatter $[5,10,11,12]$ and increasing the efficiency with which the laser energy is transferred to the target. Experiments demonstrating these latter two mechanisms are the subject of this paper and are described in the following sections. Section II discusses the transfer of energy between crossing laser beams and Section III discusses the effect of ion waves on the SRS back scatter process.

\section{Energy transfer between crossing laser beams by stimulation of ion waves}

Most designs of laser systems for inertial confinement fusion utilize multiple beams to increase the energy deposited on the target and its 
uniformity. For the indirect drive approach the laser beams are arranged so as to distribute their energy over the inside surface of the hohlraum cavity for conversion to a homogeneous flux of $x$-ray radiation while minimizing the leakage of $x$-rays through openings in the cavity. This usually means that multiple laser beams enter through each hole in the cavity at different angles. For example in a target designed for the National Ignition Facility (NIF) shown in Figure 2, ninety six separate laser beams enter though each of two laser entrance holes (LEH) in the hohlraum, with incident angles distributed uniformly in azimuth with four different polar angles, or cones. As a result there are a multitude intersecting beams pairs that can interact to stimulate plasma waves. These stimulated ion waves may scatter substantial power if they are driven resonantly by the two interacting beams [8]. In a homogeneous plasma this can occur when the familiar three wave resonance conditions and the acoustic wave dispersion relation are satisfied.

$$
\begin{aligned}
& \omega_{1}=\omega_{2}+\omega_{i a} \\
& \mathbf{k}_{1}=\mathbf{k}_{2}+\mathbf{k}_{i a} \\
& \omega_{i a}=c_{s}\left|\mathbf{k}_{i a}\right|+\mathbf{v}_{o} \bullet \mathbf{k}_{i a}
\end{aligned}
$$

Here $\omega_{1,2}$ and $k_{1,2}$ are the frequencies and wave numbers of the two intersecting beams, $c_{\mathfrak{S}}$ is the acoustic speed, and $v_{0}$ is the plasma flow velocity. When the two incident beams have the appropriate $\mathbf{k}$ and $\omega$ the amplitude of the thermally generated ion acoustic waves will grow as they propagate in space, growing fastest in the direction determined by difference of the wave vectors of the two incident beams $\left(\mathbf{k}_{1}-\mathbf{k}_{2}\right)$. This ion wave then scatters energy from beam 1 to beam 2 and can cause an amplification of the second beam and an attenuation of the first. As a result the angular distribution of the incident energy in multi-beam laser experiments can be modified by interactions between the beams. 
It is apparent from Eq. 1 that when the plasma is stationary $\left(v_{0}=0\right)$, the two incident beams must have different frequencies to be resonant, suggesting that energy transfer can occur when the lasers have a bandwidth comparable to the ion wave frequency. Because laser bandwidth is often used to suppress the plasma instabilities associated with the decay of a single beam [13], many laser systems do have some form of bandwidth in this range. In fact the NIF is presently conFigured to allow for a bandwidth of the right magnitude for energy transfer [4] with a spectral shape comprised of four separate lines separated by $1.5 \AA$ (at $351 \mathrm{~nm}$ ) [14]. However recent work [3] indicates that a continuous spectrum with a lower bandwidth of 1 to $1.7 \AA$ may be adequate to reduce the back scatter and will allow energy to be delivered to the target efficiently with a reduced possibility of resonant interaction between the beams. Although this bandwidth is low enough that resonant interactions between beams should not occur in stationary plasmas, the resonant transfer of energy between beams is still a concern because of the effects of plasma flow. It is also apparent from Eq. 1c that plasmas with flow velocities that are comparable to the sound speed (Mach 1) will allow for the formation of ion waves with zero frequency, which can result in a resonant interaction between two beams with identical frequencies or, equivalently, with low bandwidths. Because Mach 1 velocities are easily produced by the natural expansion and cooling of plasmas produced by lasers, and have been observed in indirect drive targets near the location of intersection of the beams [15], it might be supposed that energy transfer between beams has been occurring in many previous indirect drive experiments with multiple single frequency beams. A more careful inspection of the geometry of early experiments indicates that this is not likely, because the symmetry inherent in those experiments suppressed the effect. The effect of the symmetry 
derives from two sources; the single cone along which the beams are incident and the symmetry of the target about its axis so that the plasma flow must be primarily axial in the region near the axis. This results in the beams all having the same angle with respect to the flow velocity so that they all experience equal Doppler shifts. The quantity $\mathbf{v}_{0} \cdot \mathbf{k}_{1,2}$ is therefore identical for all beams in a single cone geometry and the quantity $\mathbf{v}_{\mathrm{o}} \cdot \mathbf{k}_{\text {iaw }}$ in Eq. $1 \mathrm{c}$ tends to zero, suppressing the effect of plasma flow. Although early experiments, such as those at the Nova laser facility [16], use lasers arranged in a single cone at each end of the target as shown in Figure $2 b$, it is now clear that large laser systems in the future will have beams arranged on more than one cone. In such experiments beams will cross with more than one incident angle, so that the $\mathbf{v}_{\mathrm{o}} \cdot \mathbf{k}_{\mathrm{iaw}}$ in Eq. 1c will no longer be small, and resonant excitation of ion waves can occur when the plasma flow approaches Mach 1. Hence large ion waves can be stimulated in laser systems which have large bandwidth or beams incident at multiple angles. For these reasons experiments to study the scattering and transfer of energy between beams that are caused by these ion waves have been carried out using the ignition relevant plasmas now routinely produced at Nova [2,3]. In addition to demonstrating the possibility of energy transfer between beams, such experiments also provide valuable information on the SBS side scatter mechanism in plasmas relevant to ignition experiments, such as the convective gain rate and ion wave response to the laser ponderomotive force.

In these experiments we use the recently developed capability [3] to tune the wavelength of one of ten Nova laser beams by as much as $7 \AA$ with respect to the other beams, to allow two of the beams in interact resonantly with an ion wave in a plasma that is nearly stationary $\left(v_{o} \sim 0\right)$. We observe [4] the amplification of a probe laser beam by interaction with a second frequency 
shifted beam and a stimulated ion acoustic wave. The maximum observed amplification of the long wavelength, probe laser beam is 2.8 when the frequency difference $\Delta \omega$ of the two beams is in the vicinity of the ion acoustic resonance $\left(\Delta \omega=c_{S}|\Delta k|\right.$ where $c_{S}$ is the acoustic speed and $\left.\Delta \mathbf{k}=\mathbf{k}_{1}-k_{2}\right)$. The amplification is steady state in that it persists for many $\left(\sim 10^{3}\right)$ ion acoustic periods, and is resonant in that it is largest at a particular frequency difference. Further, in the large, mixed species plasmas that are used in these experiments, we find that the amplification of the probe beam is independent of its intensity up to a maximum average scattered intensity of $1.0 \times 10^{15} \mathrm{~W} / \mathrm{cm}^{2}$. This indicates that the ion response $n_{1}$ remains unsaturated up to $n_{1} / n_{0} \sim 1 \%$.

The experiments were performed in an approximately spherical plasma [2] produced by eight $f / 4.3, \lambda=351 \mathrm{~nm}$ beams of Nova. These heater beams have a $1 \mathrm{~ns}$, square pulse shape with a total power of 20 TW (2.5 TW each beam) and pass through a $1 \mathrm{~atm} . \mathrm{C}_{5} \mathrm{H}_{12}$ gas contained in a $500 \mathrm{~nm}$ thick spherical polyimide shell with radius $\mathrm{r}_{0}=1.3 \mathrm{~mm}$. Each heater beam is centered within $180 \mu \mathrm{m}$ of the target center with a converging focus. The heater beam spot size at the target center is approximately $1.7 \mathrm{~mm}$ allowing spatially uniform heating. Two dimensional numerical simulations using LASNEX [17] indicate that after $t=400 \mathrm{ps}$ a plasma is formed which is stationary ( $\left.\mathrm{v} \leq 0.15 \mathrm{c}_{\mathrm{S}}\right)$, with a uniform density plateau $(\Delta \mathrm{n} / \mathrm{n} \leq 0.11)$ in the region $\mathrm{r} \sim 80$ to $900 \mu \mathrm{m}$. By $\mathrm{t}=1.4 \mathrm{~ns}$, the outer radius of the density plateau is reduced to approximately $r=450 \mu \mathrm{m}$ by an incoming shock wave created by the ablation of the polyimide shell. The plasma parameters calculated by the simulation, and in agreement with measurements [2] are; a density of $\mathrm{ne}=10^{21} \mathrm{~cm}^{-3}\left(0.1 \mathrm{n}_{\mathrm{Cr}}\right.$ where $\mathrm{n}_{\mathrm{Cr}}$ is the critical density for $\lambda=351 \mathrm{~nm}$ light) and electron temperature $T_{e}=3 \mathrm{keV}$. 
The high intensity pump beam and low intensity probe beam are also $f / 4.3$ and $\lambda_{\mathrm{o}}=351 \mathrm{~nm}$, and are aligned to cross at $\mathrm{r}=400 \mu \mathrm{m}$ near best focus for both beams. As a result, the interaction between the two electromagnetic waves and an ion acoustic wave occurs in the plateau region of the plasma as shown in Figure 3. The interaction beams have random phase plates (RPPs) which smooth the intensity profile and limit the full width at half the maximum spot size to $177 \mu \mathrm{m}$ ( $345 \mu \mathrm{m}$ between first Airy minima) and the peak pump intensity to $1 \times 1016 \mathrm{~W} / \mathrm{cm}^{2}\left(2 \times 1015 \mathrm{~W} / \mathrm{cm}^{2}\right.$ average inside the Airy minima) in vacuum. The beams have their polarizations aligned within $25^{\circ}$ of parallel. The simulations indicate that the presence of the focused probe beam increases the temperature only slightly $(\sim 6 \%)$ in the vicinity of best focus. The probe beam is adjusted to have a wavelength slightly longer than that of the pump with the wavelength separation ranging between 0.0 and $0.73 \mathrm{~nm}$. The probe beam has a square pulse shape lasting $2.0 \mathrm{~ns}$ which begins at the same time as the heaters $(t=0)$. The pump beam has a $1.0 \mathrm{~ns}$ square pulse shape that is delayed $0.4 \mathrm{~ns}$ so that the plasma in the interaction region is relatively homogeneous during the interaction period. The transmitted power of the probe beam is collected by a fused silica plate $1.5 \mathrm{~m}$ from the target, which is roughened to scatter the light over a broad angle [18]. This scattered light is imaged onto a fast photo diode and a gated optical imager. The photo diode is calibrated to provide a time history of the probe beam power which is transmitted through the plasma and onto the scatter plate. The imager captures a 2-D image of the light on the scatter plate during the $0.6 \mathrm{~ns}$ period when all beams are on and provides a measure of the directionality of the transmitted power during the period of the interaction. 
As discussed in Section I, at the intersection of the pump and probe beam, an ion wave forms in response to the ponderomotive force determined by the product of the electric fields of the two incident beams. As a result the initial ion wave amplitude is then proportional to the probe beam incident amplitude. It follows that, the energy scattered from the pump is also proportional to the probe intensity, and an amplification of the probe results. This amplification is defined to be the ratio of the transmitted power measured in an experiment with the pump beam on, to that measured in an essentially identical experiment with the pump beam off. The transmitted power waveforms with and without a pump beam are shown in Figure 4, for the case of $\Delta \lambda=0.43 \mathrm{~nm}$, and a normalized probe intensity of $\mathrm{I}_{\text {probe }} / \mathrm{I}_{\text {pump }}=0.06$. The total transmitted energy without a pump beam is $49 \pm 7 \%$ of the incident energy which compares well with a calculation of inverse bremsstrahlung absorption and refraction by the plasma [18]. This transmission is reproducible within $\pm 15 \%$. The shape of the waveform in the pump-off case in Figure 4 shows that the transmission first increases in time when the plasma temperature is increasing and then decreases in time after the heaters turn off at $1.0 \mathrm{~ns}$ and the plasma cools by expansion. Comparing the 'pump on' waveform in Figure 4 to the 'pump off' shows an increase in the probe's transmitted power during the time the pump beam is on $(0.4 \leq t \leq 1.4 \mathrm{~ns})$. The 'pump on' waveform is corrected to account for a small variation in the energy of the probe beam between the two shots. The transmitted probe power is nearly the same in the two experiments before the arrival of the pump. After $\mathrm{t}=0.4 \mathrm{~ns}$, the transmitted probe power rises in about $150 \mathrm{ps}$ to nearly 1.7 times the level observed without the pump indicating amplification of the probe by the pump. After the pump turns off the probe power drops rapidly to equal the value observed without the pump. The 
amplification is determined from the ratio of the two traces and is found to vary between 1.6 and 1.8 during the 1 ns duration of the pump pulse. The average amplification $A$ is determined by averaging over the $1 \mathrm{~ns}$ that the pump is on plus an additional $0.3 \mathrm{~ns}$ to include signal delayed by the response of the detector, and is found to be 1.7 in this case.

The amplification observed in Figure 4 can be explained by scattering from an ion wave produced by the beating of the incident and pump beams. Resonant interaction requires that this ion wave have a wave vector $k_{i a}=\Delta k$ $=\mathbf{k}_{1}-\mathbf{k}_{2}$. Experimental evidence of the wave number matching is obtained from the 2-D image of the transmitted probe beam on the scatter plate as described in Ref [18]. The amplified beam is largest inside the $f / 4.3$ cone of the incident beam $\left( \pm 6.6^{\circ}\right)$ and similar to the profile of the probe beam obtained when it is transmitted through the plasma with the pump beam off. The intensity of the amplified beam falls rapidly outside of the $f / 4.3$ cone (FWHM $=12^{\circ}$ ) indicating that the scattering is caused by ion waves with wave numbers near the matched value $\Delta \mathbf{k}$.

Experiments to determine the average energy amplification as a function of the frequency mismatch were performed for six different wavelength separations between 0.0 and $0.73 \mathrm{~nm}$ with the normalized probe intensity $I_{\text {probe }} / I_{\text {pump }}$ between 0.06 and 0.32 . The amplifications for these cases are shown in Figure 5 and exhibit significant gain only when there is a frequency separation between the two beams. The largest amplification is 2.8 when the wavelength separation is $\Delta \lambda=0.58 \mathrm{~nm}$. The resonant ion wave frequency is calculated as $\omega_{i a}=c_{S}|\Delta k|$ and the half width of the resonance as $v_{i} / 2$, where $v_{i}$ is the intensity damping rate of the ion wave $[4,8]$. The predicted position of the resonance is $\Delta \lambda=0.46 \mathrm{~nm}$ with a width of $\pm 0.04 \mathrm{~nm}$ for the plasma parameters found in the vicinity of the focused beam. This 
indicates that the observed maximum measured gain is near the ion wave resonance, but may be Doppler shifted by weak plasma flows produced by heater beam and target inhomogenities that are not included in the 2D simulations. The observation of a maximum amplification near the ion wave frequency combined with the observation of reduced amplification at both larger and smaller frequencies $(\Delta \lambda=0.3 \mathrm{~nm}$ and $0.73 \mathrm{~nm})$ indicates that resonant excitation of an ion acoustic wave is necessary for amplification.

To compare with theory, we adopt the model of a steady state, convective instability which leads to the amplification A of the probe by a gain $G$ such that $G=\ln (A)$. This interpretation is applied in this case because the beam crossing angle is less than 90 degrees (forward scattering) so that the instability cannot be absolute, and because the duration of the experiment ( 1 ns) is long compared to the time to reach a steady state, which, in the strong damping limit is the ion wave damping time $(\sim 1.0 \mathrm{ps})$. In this limit the gain exponent in a homogeneous plasma scales as $\mathrm{nLI}_{\text {pump }} /\left(\mathrm{T}_{\mathrm{e}} \mathrm{v}_{\mathrm{i}}\right)[8]$ where $\mathrm{L}$ is the length of the interaction region. The effect of the pump wave is to scatter its power in the direction of the probe wave such that the transmitted probe wave amplitude is proportional to both the incident probe wave amplitude and an exponential of the pump wave intensity. The measured amplification is also affected by small scale inhomogeneities both in the incident beam [19] and in the plasma [8], for which accurate characterization is difficult. These inhomogeneities make a calculation of the gain of the instability based on the simple model of coherent laser beams in uniform plasmas an over estimate of the actual gain. In fact, under the conditions of this experiment, the ideal model indicates a gain of $G \sim 20$ when the probe beam perfectly tuned $[8,20,21]$ while the maximum observed gain is $G=1.0$. These values can be substantially reconciled by considering calculations 
including the two types of inhomogeneities and recognizing that the set of discrete measurements in Figure 5 may miss the exact resonance and underestimate the peak gain $[5,8]$.

To investigate the dependence of the scattered power on the incident probe power, experiments were performed at both high and low scattered power at the frequency separation of maximum observed gain, $\Delta \lambda=0.58 \mathrm{~nm}$. The high probe intensity was $\mathrm{I}_{\text {probe }} / \mathrm{I}_{\text {pump }}=0.32$ and the low intensity was $I_{\text {probe }} / I_{\text {pump }}=0.13$. The measured amplification of the probe at high intensity was $2.8 \pm 0.42$ and at low intensity was $2.5 \pm 0.37$, indicating that the gain is nearly independent of the probe intensity up to this level and that the ion wave is unsaturated. Under these conditions a maximum of $52 \% \pm 12 \%$ of the pump energy was transferred to the probe. This large an energy transfer should lead to a $23 \%$ reduction in the spatially averaged amplification due to pump depletion. This effect was not observed but it is comparable to the combined shot to shot variation in the transmission coefficient $( \pm 15 \%)$ and amplification in these measurements. The amplitude of the ion wave is estimated by considering the amplitude necessary to scatter $1 / 2$ the probe energy in a volume of dimension $L$, according to the formula $n_{1} / n_{0}=$ $(2 / \pi)(2)^{1 / 2}\left(\omega / \omega_{p}\right)^{2}\left(\lambda_{0} / L\right)$. A lower limit to the maximum ion wave amplitude observed in this experiment is $n_{1} / n_{0}=1 \%$, assuming the scattering occurs uniformly over the region in which the beams intersect $(\mathrm{L}=345 \mu \mathrm{m})$. The actual wave amplitude may be higher if inhomogeneities localize the scatter to a region smaller than $L$. The data in Figure 5 indicates a linear dependence of transmitted power on the incident probe power over a wide range if the experiments done at $\Delta \lambda=0.45 \mathrm{~nm}$ and $0.6 \mathrm{~nm}$ are considered. The transmitted power measured with values of $I_{\text {probe }} / I_{\text {pump }}$ different than 0.13 are normalized to the transmitted power measured at the same $\Delta \lambda$ but with 
$I_{\text {probe }} / I_{\text {pump }}=0.13$. These normalized values are plotted in Figure 6 and show that the transmitted power is proportional to the incident probe power over a range of 5:1, in good agreement with Ref. [8].

These experiments demonstrate that substantial energy can be transferred between two laser beams in a plasma with properties similar to what is expected in ignition experiments, provided the conditions for resonance (Eq. 1a,b,c) are. satisfied. The measurements have further revealed that there is no saturation mechanism operating in these plasmas even when ion wave amplitudes are large enough to transfer as much as $50 \%$ of the incident beam energy to the second beam, and that the energy transfer mechanism is strongly sensitive to the frequency mis-match between the laser beams (ie; resonant) so that frequency tuning of the incident beams may be used to control the energy transfer.

III Moderation of SRS driven Langmuir waves by the stimulation of ion waves

In addition to the direct effect that ion waves have on the transport of electromagnetic waves via the SBS process, ion waves can also have an indirect effect by moderating the SRS process. In the three wave SRS process a Langmuir wave grows from noise and scatters the incident electromagnetic wave. The scattered electromagnetic wave and the incident wave beat to produce a ponderomotive force field with wave number and frequency matched to the scattering Langmuir wave. As a result the Langmuir wave and the scattering both grow in space and time until they are saturated by some other process. Because the Langmuir waves and scattering produced by SRS have been found to be responsible for much of the scattered energy in ignition scale plasmas $[3,19,22]$ the mechanisms for their saturation are of great interest $[5,10,11,12,19]$. The simplest mechanism for saturation is the 
convection of energy out of the illuminated plasma volume by the scattered electromagnetic wave, or convective saturation. When the SRS process grows to the linear convective saturation level in a large scale length plasma (ie; in the strong damping limit) the scattered power is exponentially dependent on the inverse of the Langmuir wave damping rate. Recent calculations of the Landau damping rate of Langmuir waves [19] show that deviations of the electron distribution function from the Maxwellian form that occur when the plasma is illuminated by a high intensity laser [23] cause the damping rate to be very small under conditions relevant to ignition experiments, and in fact, to approach the minimum set by collisional damping in a laser hot spot in a high $Z$ plasma. As a result substantial energy could be scattered by SRS in the gold plasma formed near the wall of the targets used in ignition experiments if convection is the only saturation mechanism operating. However observations [3,22] indicate that SRS is well below the convective saturation limit and is mediated by a process involving ion waves $[5,12]$.

The strongest evidence that SRS will be mediated by ion waves in ignition experiments comes from experiments in plasmas that simulate the conditions of ignition experiments and in which the ion wave damping is varied while other plasma conditions are constant. The results show a strong dependence of the energy scattered by SRS on the damping rate of the ion waves. These experiments were performed with gas filled Au hohlraum targets which provide plasma density, electron temperature, and profile, shown in Figure 7, which is comparable to what is expected in ignition experiments [3]. The important difference between the experiments described here and those done previously [3] is that a region of the Au wall is impregnated with a Be impurity. The Be impurity has little effect on the x-ray 
properties of the wall material and therefore has little effect on the radiation and plasma conditions present in the hohlraum. What the Be impurity primarily affects is the number of particles present with velocities that match the phase velocity of the ion acoustic wave. Varying the fraction of Be impurity therefore allows the Landau damping rate of ion acoustic waves to be varied experimentally. The target is fabricated as a pure Au cylindrical hohlraum (1.6 mm dia. $\times 2.75 \mathrm{~mm}$ ) with 25 micron wall thickness with a 1.2 $\mathrm{mm}$ dia. laser entrance hole (LEH) at each end to allow the ten Nova laser beams to illuminate the inside of the cylinder. A $1.35 \mathrm{~mm} \times 0.94 \mathrm{~mm}$ rectangular hole is cut in the wall at the location at which the probe beam is pointed and covered with a $\mathrm{Au} / \mathrm{Be}$ patch. The LEH's are covered with $3500 \AA$ polyimide film so that the hohlraum can be filled with a low $\mathrm{Z}$ gas. The $\mathrm{Au} / \mathrm{Be}$ patch is fabricated with layers of Au alternated with layers of Be deposited on an Au substrate where the relative thickness of the two layers is varied to control the fraction of Be. The thickness of a layer pair is $110 \AA$ and the total thickness of the layered region is either 2.6 or 5 microns (experiments with the two different thicknesses showed no significant difference in the reflected light). In these targets the plasma formed in the path of the probe beam has a variable fraction of Be. Calculations of the ion wave response in a plasma with multiple atomic species [20,21] shows that the damping rate is proportional to the fraction of Be down to about $1 \%$ of the ion acoustic frequency at which point the dominant damping mechanisms changes from ion Landau damping to electron Landau damping. Damping rates of up $24 \%$ are obtainable with an atomic fraction of $\mathrm{Be}$ of $80 \%$, moreover atomic fractions below $50 \%$ make little change in the real part of the dispersion relation while maintaining damping rate approximately proportional to the impurity concentration. 
The hohlraum is illuminated with ten beams of $351 \mathrm{~nm}$ light that pass through one of two laser entrance holes on either end of the cylindrical cavity and are incident on the interior surface of the cavity as shown in figure $2 b$. The incident laser beams have a $2.5 \mathrm{~ns}$ pulse that is temporally shaped so that the intensity at the wall ramps up to $2 \times 10^{15} \mathrm{~W} / \mathrm{cm}^{2}$ between 1 and $2 \mathrm{~ns}$ (P.S. 22) as described in Ref. [3]. The beam which is incident on the $\mathrm{Au} / \mathrm{Be}$ patch has various types of beam smoothing applied including; random phase plate smoothing only (RPP), and RPP smoothing with $1 \AA$ bandwidth in addition ( $1 \AA$ at $3 \omega$, or equivalently $3 \AA$ at $1 \omega$ ) that is spatially dispersed across the lens in the near field (SSD, [13]) using either a 1200 line $/ \mathrm{mm}$ grating or a 1700 line $/ \mathrm{mm}$ grating. The effect of bandwidth dispersed across the lens is to reduce the coherence time of laser hot spots at the target plane to the inverse of the bandwidth. The effect of the grating is to disperse all of the bandwidth across the lens in the case of the $17001 / \mathrm{mm}$ grating, or to disperse only approximately $2 / 3$ of the available bandwidth across the lens in the case of the $1200 \mathrm{l} / \mathrm{mm}$ grating. The uniformity of the spatial intensity profile of the beam is increased as 'beam smoothing' is increased from; 'unsmoothed' to 'RPP only' to 'RPP plus $1200 \mathrm{l} / \mathrm{mm}$ dispersion' to 'RPP plus $1700 \mathrm{l} / \mathrm{mm}$ dispersion'. The spectrum and time history of the light scattered from the smoothed beam within $20^{\circ}$ of direct back scatter (including both light scattered into the lens and light scattered just outside the lens cone) is measured with two spectrometers as shown in Figure 8 in the case of $20 \%$ Be in the wall. Figure 8 shows the measurements taken with two spectrometers, one viewing wavelengths close to the incident wavelength (SBS) and another viewing wavelengths shifted to the visible (SRS) $[3,7]$ Experiments were performed with Be concentration of $0 \%, 20 \%, 50 \%$ and $80 \%$ and various types of beam smoothing. The temporally and spectrally integrated SBS and SRS 
reflectivities measured in both $\mathrm{CH}_{4}$ and $\mathrm{C}_{3} \mathrm{H}_{8}$ filled hohlraums as a function of beam smoothing are shown in Figures 9 and 10. Figures 9 and 10 show a strong dependence of the SRS on the concentration of Be for a variety of smoothing conditions, and a somewhat weaker dependence on beam smoothing with the lowest reflectivities occurring at $0 \%$ Be and maximum beam smoothing (RPP+ SSD with $1700 \mathrm{l} / \mathrm{mm})$. The concentration of $\mathrm{Be}$ is interpreted as ion wave damping using the electron and ion temperatures calculated by LASNEX [17] $\left(\mathrm{T}_{\mathrm{i}} / \mathrm{T}_{\mathrm{e}}=0.7\right)$ and is shown on the upper axis in Figures 9 and 10, demonstrating that SRS increases strongly with ion wave damping while the SBS decreases only slightly. The former result indicates that SRS is being mediated by a process involving ion waves while the latter is qualitatively consistent with three wave SBS occurring in an inhomogeneous plasma with strong damping of the ion wave [24]. Further, the magnitude of the reflectivity due to both SRS and SBS is lower than predicted by convective saturation theory as discussed in reference [3]. Figures 9 and 10 also show that the RPP beam smoothing is most effective at reducing SBS with the $f / 4.3$ beam used in these experiments.

To further understand the mechanism by which SRS is dependent on ion wave damping a second series of experiments has been performed using the gas bag target described in Section I to produce plasmas that are similar to those in hohlraum experiments but that are much more uniform and easier to characterize than hohlraums. In these experiments the gas bag is filled with Xe with a $\mathrm{C}_{5} \mathrm{H}_{12}$ impurity to control the ion wave damping. LASNEX simulations [5] show that the Xe gas ionizes to a charge state of about 40 just as the Au does in the hohlraum plasmas, and therefore has very similar collisional properties, while the $\mathrm{C}_{5} \mathrm{H}_{12}$ fully ionizes to produce a similar number of electrons per molecule (42) to the Xe. As a result the fraction of 
$\mathrm{C}_{5} \mathrm{H}_{12}$ impurity can be varied between 0 and $30 \%$ without affecting the electron density significantly, while variations of the impurity concentration in this range are found to change the ion wave damping by a ratio of 15:1. Moreover measurements of x-ray spectra and transmitted light indicate that the presence of the impurity in concentrations that are less than $30 \%$ in these plasmas makes an insignificant perturbation to the collisional absorption rate, electron temperature, or radiation properties, so that any measured dependence of the reflectivities on impurity concentration can be interpreted as a dependence on ion wave damping.

The experiments were carried out using gas bag targets, as described in Section $\mathrm{I}$, that are filled with the $\mathrm{Xe} / \mathrm{C}_{5} \mathrm{H}_{12}$ mixture. With this material the electrons are heated to a temperature of $T_{e}=3.6 \mathrm{keV}$ during the last half of the square heater pulse ( $t=0.5$ to $1.0 \mathrm{~ns}$ ) when the plasma parameters are most constant in space and time $[2,5]$. The electron density is $n_{e}=8.5 \times 10^{20}$ $\mathrm{cm}^{-3}$, determined by the initial gas density and ionization state. The temperature is determined from measurements of $x$-ray line ratios and $x$-ray transport modeling and is in agreement with LASNEX [17] simulations. The measured transmission of a beam through the plasma [18] varies only from $1 \%$ to $2 \%$ when the impurity concentration is varied from $0 \%$ to $30 \%$ and is also in agreement with the simulations, indicating that the electron temperature variations are small $(\leq \pm 6 \%)$. Measurements of the radiated $x$ ray power in the photon energy range of 0.2 to $2 \mathrm{keV}$ indicate that the radiated power is also constant within $\pm 15 \%$ over the same range of impurity concentration. The lack of dependence of the electron density and temperature on impurity concentration indicates that the frequency and damping rate of the Langmuir wave are also constant. 
An interaction beam, produces 1.5 TW continuously for $1.0 \mathrm{~ns}$ with $\lambda=$ $351 \mathrm{~nm}$. This beam is delayed $0.5 \mathrm{~ns}$ with respect to the heaters and focused at the plasma center. Reflectivity measurements are made during the 0.5 to 1.0 ns (early) period when the heaters are on, as well as during the 1.0 to $1.5 \mathrm{~ns}$ (late) period when the plasma is cooling and less homogeneous. The interaction beam is smoothed by a random phase plate (RPP) and $1 \AA \mathrm{FM}$ bandwidth dispersed across the beam with the $1200 \mathrm{l} / \mathrm{mm}$ grating, so that its peak intensity and spot size in vacuum are $7.0 \times 10^{15} \mathrm{~W} / \mathrm{cm}^{2}$ and $177 \mu \mathrm{m}$ FWHM (345 $\mu \mathrm{m}$ between first Airy minima). The plasma properties encountered by the interaction beam during the early period are calculated by LASNEX [17] for a $90 \%$ Xe, $10 \% \mathrm{C}_{5} \mathrm{H} 12$ gas mix, indicating a temperature, and density plateau near the plasma edge as shown in Figure 11. The classical collisional absorption length for $351 \mathrm{~nm}$ light in Xe with a $3.6 \mathrm{keV}$ electron temperature and $8.5 \times 10^{20} \mathrm{~cm}^{-3}$ electron density, is $800 \mu \mathrm{m}$. Therefore, the majority of the back scattering occurs outside $r=0.5 \mathrm{~mm}$. The down shifted light scattered within $20^{\circ}$ of direct back scatter is measured [7] with a streaked optical spectrometer in the visible, with a spectral range of 400 to $700 \mathrm{~nm}$, and a second spectrometer in the UV, with a spectral range of 346 to $361 \mathrm{~nm}$. The case of $\mathrm{Xe}$ with $5.5 \% \mathrm{C}_{5} \mathrm{H}_{12}$ impurity is shown in Figure 12 . During the early period the peak of the spectrum is at $575 \mathrm{~nm}$ consistent with scattering from a Langmuir wave propagating in a plasma with a density equal to $10 \%$ of the critical density and a $3.0 \mathrm{keV}$ electron temperature comparable to the simulated plasma parameters near $r=1.2 \mathrm{~mm}$. After the heaters turn off at $1.0 \mathrm{~ns}$ the peak shifts to the blue indicating the cooling and expansion of the plasma.

Experiments were performed with six different impurity concentrations between $0 \%$ and $30 \% \mathrm{C}_{5} \mathrm{H}_{12}$ and exhibit a strong dependence 
of the SRS reflectivity on the concentration of $\mathrm{C}_{5} \mathrm{H}_{12}$ which is interpreted as a dependence of Langmuir wave amplitude on the damping rate of the ion acoustic wave. The integrated energies from the two time periods are expressed as percent reflectivities of the incident beam power due to SRS and plotted in Figure 13. In the integrated data it is clear that late time SRS reflectivities are approximately proportional to the impurity concentration for all concentrations studied. The early time reflectivities are proportional to concentration up to $10 \%$ and become independent, or a mildly decreasing function, of concentration between 10 and $30 \%$. Because the ion acoustic damping rate is expected to be linear with impurity concentration in this case $[20,21]$ a linear dependence of reflectivity on impurity concentration is interpreted as a linear dependence on the damping of the ion acoustic wave. The possibility that dependence of the reflectivity on ion wave damping is actually the result of suppression of SRS by large amplitude ion waves generated by SBS is ruled out by using the SBS reflectivity as a measure of the amplitude of the ion waves. As discussed in Ref. [5] the SBS does not monotonically decrease with ion acoustic damping indicating that the increase in SRS observed in Figure 13 cannot be due a reduction of ion wave amplitude and therefore a reduction of the detuning effect that ion waves can have on SRS. On the contrary the SBS is constant or increasing with ion wave damping in some of the cases were SRS is also increasing. These observations demonstration of the direct dependence of SRS reflectivity on the damping rate of the ion acoustic wave.

A simple model is used to show how the observed dependence of SRS on ion wave damping may be consistent with Langmuir waves that are undergoing a secondary three wave decay involving ion waves. In this model the intensity profile of the beam and filamentation are neglected for 
simplicity, and the Langmuir wave amplitude is assumed to be in the vicinity of the thresholds for the secondary decay via the instabilities discussed in Section I. These thresholds for decay, expressed in terms of the density fluctuation, are given by Ref. [10] for LDI and by Ref. [25] for EDI.

$$
\begin{aligned}
& \left(\frac{\delta \mathrm{n}}{\mathrm{n}}\right)_{\mathrm{EDI}}=4 \mathrm{k}_{\mathrm{L}} \lambda_{\mathrm{D}}\left(\frac{v_{\mathrm{ia}}}{\omega_{\mathrm{ia}}}\right)^{1 / 2}\left(\frac{v_{\mathrm{em}}}{\omega_{\mathrm{p}}}\right)^{1 / 2} . \\
& \left(\frac{\delta \mathrm{n}}{\mathrm{n}}\right)_{\mathrm{LDI}}=4 \mathrm{k}_{\mathrm{L}} \lambda_{\mathrm{D}}\left(\frac{v_{\mathrm{ia}}}{\omega_{\mathrm{ia}}}\right)^{1 / 2}\left(\frac{v_{\mathrm{L}}}{\omega_{\mathrm{p}}}\right)^{1 / 2}
\end{aligned}
$$

Where $k_{\mathrm{L}}$ is the wave vector of the SRS generated Langmuir wave, $v_{\mathrm{ia}} / \omega_{\mathrm{ia}}$ is the normalized linear ion wave damping rate, $v_{\mathrm{em}}$ is the linear amplitude damping rate of the secondary electromagnetic wave and $v_{\mathrm{L}}$ is the linear amplitude damping rate of the secondary Langmuir wave, and $\lambda_{D}$ is the electron Debye length and $\omega_{\mathrm{p}}$ is the plasma frequency. The measured SRS light is interpreted as Thomson scattering off density fluctuations in the scattering volume as described in Ref [5]. A lower limit to the Langmuir wave amplitude is determined using an estimate for the correlation length of the waves determined from the width of the scattered spectrum. The reflectivity shown on the left axis of Figure 14 indicates a wave amplitude $\delta n / n$ shown on the right axis. When applied to the measurements this model indicates that Langmuir wave amplitudes are proportional to the ion wave damping and comparible to the thresholds for secondary decay that are estimated from the early time plasma conditions and shown as lines in Figure 13. The linear dependence is most clearly observed in the reflectivity data taken at early time when the impurity concentration is less than $10 \%$. For impurity concentrations above $10 \%$ the early time reflectivity is not very 
dependent on the ion wave damping and falls below the thresholds for the secondary decay. This is likely due the convective saturation of the SRS generated Langmuir wave before it reaches the secondary decay threshold. The secondary decay mechanism will only determine the SRS reflectivity when the primary three wave process is sufficiently strong to drive the Langmuir wave amplitude to the threshold for the secondary decay. Thus at early time and high impurity concentration $(>10 \%)$ the secondary decay threshold is sufficiently high that the SRS reflectivity is likely limited by the convective saturation of the three wave SRS process and is therefore observed to be approximately independent of ion wave damping. The threshold for the EDI instability is reached using Langmuir wave amplitudes that are averaged over the entire beam profile, however as described in Ref. [4] and [23] the LDI threshold is strongly intensity dependent in high $\mathrm{Z}$ plasmas and wave amplitudes may easily be above threshold in hot spots of the beam. The late time data show linear dependence of the reflectivity on the impurity concentration up to much higher concentration than at early time. This may be because at late time the plasma has cooled and therefore has a higher convective saturation level for the three wave process consistent with the reduced Landau damping of the Langmuir wave at low $T_{e}$. A high convective saturation level allows the Langmuir waves to grow to the secondary decay threshold even when ion wave damping is large, resulting in reflectivities that follow the linear scaling with ion wave damping up to at least $30 \%$, as shown in Figure 13. However quantitative analysis of the late time data is complicated by variations of the plasma properties in time and space.

The experiments with hohlraums indicate a dependence of the SRS reflectivity on ion wave damping in plasmas with conditions similar to those 
expected in the NIF. The experiments with gas bags show that in homogeneous plasmas with otherwise comparable conditions the amplitude of the Langmuir wave is large enough to undergo a secondary decay involving an ion wave, and in fact tracks the threshold for such a decay, maintaining the amplitude to be approximately proportional to ion wave damping.

\section{Discussion and conclusions}

In conclusion it has been shown by experimental demonstration that there are at least three mechanisms by which ion waves are likely to be stimulated in ignition scale experiments, each of which may affect the energy delivered to the target or its angular distribution. The mechanisms for generating ion waves are; stimulation by multiple crossing laser beams, stimulation by the decay of large amplitude Langmuir waves produced by the decay of the individual laser beams, as well as the widely studied single laser beam process of stimulated Brillouin scattering (SBS). The multi-beam mechanism has been demonstrated in experiments with frequency shifted beams where it is shown that energy can be transferred between laser beams, with maximum energy transferred when the difference in the beams frequencies is in the vicinity of the frequency of the ion acoustic wave. The stimulation by Langmuir waves is indicated by the observation that the amplitude of the Langmuir wave is in the vicinity of the threshold for stimulation of ion waves by either the LDI and EDI mechanism, and the amplitude of, and therefore the scattering by the Langmuir waves is limited to a value that is dependent on ion wave damping. Because the energy transfer between beams is found to be strongly affected by small changes in the frequency of the interaction beams it is controllable by careful design of 
the laser bandwidth and the plasma flows available for Doppler shifting the beams. The primary effect of the decay of the Langmuir waves is to reduce the energy scattered by stimulated Raman scattering (SRS) thereby increasing efficiency, as well as to make the scattering dependent on the damping rate of the ion waves. As a result the SRS reflectivity in mixed species plasmas can be controlled by adjusting the fraction of high and low $\mathrm{Z}$ components. 


\section{References}

[1] J. Lindl, Phys. of Plasmas 2, 3933 (1995).

[2] S. H. Glenzer, C. A. Back, K. G. Estabrook, B. J. MacGowan, D. S. Montgomery, R. K. Kirkwood, J. D. Moody, D. H. Munro, and G. F. Stone, Phys. Rev. E 55, 1 (1997).

[3] B. J. MacGowan, B. B. Afeyan, C. A. Back, R. L. Berger, G. Bonnaud, M. Casanova, B. I. Cohen, D. E. Desenne, D. F. BuBois, A. G. Dulier, K. G. Estabrook, J. C. Fernandez, S. H. Glenzer, D. E. Hinkel, T. B. Kaiser, D. H. Kalantar, R. L. Kauffman, R. K. Kirkwood, W. L. Kruer, A. B. Langdon, B. F. Lasinski, D. S. Montgomery, J. D. Moody, D. H. Munro, L. V. Powers, H. A. Rose, C. Rousseaux, R. E. Turner, B. H. Wilde, S. C. Wilks, and E. A. Williams Phys. Plasmas 3, 2029 (1996).

[4] R. K. Kirkwood, B. B. Afeyan, W. L. Kruer, B. J. MacGowan, J. D. Moody, D. S. Montgomery, D. M. Pennington, T. L. Weiland, and S. C. Wilks, Phys. Rev. Lett. 76, 2065 (1996).

[5] R. K. Kirkwood, B. J. MacGowan, D. S. Montgomery, B. B. Afeyan, W. L. Kruer, J. D. Moody, K. G. Estabrook, C. A. Back, S. H. Glenzer, M. A. Blain, E. A. Williams, R. L. Berger, and B. F. Lasinski, Phys. Rev. Lett. 77, 2706 (1996).

[6] L. V. Powers, R. E. Turner, R. L. Kauffman, R. L. Berger, P. Amendt, C. A. Back, T. P. Bernat, S. N. Dixit, D. Eimerl, J. A. Harte, M. A. Henesian, D. H. Kalantar, B. F. Lasinski, B. J. MacGowan, D. S. Montgomery, D. H. Munro, D. M. Pennington, T. D. Shepard, G. F. Stone, L. J. Suter, and E. A. Williams Phys. Rev. Lett. 74, 2957 (1995).

[7] R. K. Kirkwood, C. A. Back, M. A. Blain, D. E. Desenne, A. G. Dulieu, S. H. Glenzer, B. J. MacGowan, D. S. Montgomery, and J. D. Moody, to be published in Rev. Sci. Inst. Jan (1997).

[8] W. L. Kruer, S. C. Wilks, B. B. Afeyan, and R. K. Kirkwood, Phys. of Plasmas 3, 382 (1996).

[9] C. J. McKinstrie, J. S. Li, R. E. Giacone, and H. X. Vu, Phys. of Plasmas 3, 2686 (1996).

[10] J. A. Heikkinen and S. J. Karttunen, Phys. Fluids 29, 1291 (1986).

[11] B. Bezzerides, D. F. DuBois, and H. A. Rose, Phys. Rev. Lett. 70, 2569 (1993).

[12] J. C. Fernandez, J. A. Cobble, Bruce H. Failor, Donald F. DuBois, David S. Montgomery, Harvey A. Rose, Hoanh X. Vu, Bernhard H. Wilde, Mark D. Wilke, and Robert E. Chrien, Phys. Rev. Lett. 77, 2702 (1996).

[13] S. Skupsky, R. W. Short, T. Kessler, R. S. Craxton, S. Letzring, and J. M. Soures, J. Appl. Phys. 66, 3456 (1989).

[14] S. W. Haan, Stephen M. Pollain, John D. Lindl, Laurance J. Suter, Richard L. Berger, Linda V. Powers, W. Edward Alley, Peter A. Amendt, John A. Futterman, W. Kirk Levedahl, Mordecai D. Rosen, Dana P. Rowley, Richard A. Sacks, Aleksei I. Shestakov, George L. Strobel, Max Tabak, Stephen V. Wever, George B. Zimmerman, William J. Krauser, Douglas C. Wilson, Stephen V. Coggeshall, Bavid B. Harris, Nelson M. Hoffman, and Bernhard H. Wilde, Phys. of Plasmas 2, 2480 (1995) or D. M. Pennington, T. L. Weiland, M. A. Henesian, D. Eimerl, R. B. Wilcox, 
Four- Color Laser Irradiation System for Laser-Plasma Interaction Experiments, Lawrence Livermore National Laboratory, Livermore, CA, UCRL-LR-105821-95-2 (1995).

[15] S. H. Glenzer, private communication.

[16] see for example E. M. Campbell, J. T. Hunt, E. S. Bliss, D. R. Speck, and R. P. Drake, Rev. Sci. Instrum. 57, 2101 (1986).

[17] G. Zimmerman and W. Kruer, Comments in Plasma Phys. and Controlled Fusion 2, 85 (1975).

[18] J. D. Moody, B. J. MacGowan, D. H. Munro, R. L. Berger, R. K. Kirkwood, and D. S. Montgomery, Bull. Am. Phys. Soc. 39, 1753 (1994).

[19] B. B. Afeyan, A. E. Chou, and W. L. Kruer, submitted to Phy. Rev. Lett.

[20] E. A. Williams, R. L. Berger, R. P. Drake, A. M. Rubenchik, and B. S. Bauer, D. D. Meyerhofer, A. C. Gaeris, T. W. Johnston, Phys. of Plasmas 2, 129 (1995).

[21] H. X. Vu, J. M. Wallace, and B. Bezzerides, Phys. of Plasmas 1, 3542 (1994).

[22] D. S. Montgomery, B. B. Afeyan, B. J. MacGowan, C. A. Back, S. H. Glenzer, R. K. Kirkwood, J. D. Moody, G. F. Stone, R. L. Berger, K. G. Estabrook, W. L. Kruer, B. F. Lasinski, D. H. Munro, submitted to Phys. Rev. Lett.

[23] P. Alaterre, J.-P. Matte, and M. Lamoureaux, Phys. Rev. A 34, 1578 (1986);

A. B. Langdon, Phys. Rev. Lett. 44, 575 (1980).

[24] R. L. Berger, E. A. Williams, and A. Simon, Phys. Fluids B 1, 414 (1989).

[25] K. L. Baker, Ph.D. thesis, University of California, Davis (1996), see also P. K. Shukla, M. Y. Yu, M. Mohan, R. K. Varma, and K. H. Spatschek Phys. Rev. A 27, 552 (1983).

[26] T. Kolber, W. Rozmus, and V. T. Tikhonchuk, Phys. Plasmas 2, 256 (1995). 


\section{Figures}

Figure 1) Mechanisms for the stimulation of ion waves in ignition relevant plasmas include a) stimulated Brillouin scattering (SBS) from a single beam in which the incident electromagnetic wave decays into an ion wave and a scattered electromagnetic wave, b) seeding of the SBS side scatter instability by a crossing laser beam with a mismatched frequency resulting in energy transfer between the beams, and c) the decay of the Langmuir wave produced by stimulated Raman scattering (SRS), into an ion wave and a second wave.

Figure 2) a) NIF target and beam arrangement showing 192 beams with 96 beams entering a hohlraum through each of two laser entrance holes (LEH) with four different polar angles or cones.

b) Standard target and beam arrangement for Nova hohlraum experiments showing all beams entering with the same polar angle.

Figure 3) Experimental configuration showing an approximately spherical plasma produced by a gas bag target and two interaction beams crossing in a homogeneous region off the plasma center. The two beams are detuned by $\Delta \lambda=0.0$ to $0.73 \mathrm{~nm}$ to excite the ion acoustic resonance. The transmitted power vs. time of the long wavelength probe beam is measured.

Figure 4) Measurement of the probe beam power transmitted through the 
plasma for the case $\Delta \lambda=0.45 \mathrm{~nm}$ and $\mathrm{I}_{\text {probe }} / \mathrm{I}_{\text {pump }}=0.06$. In the 'pump on' case a $2 \times 10^{15} \mathrm{~W} / \mathrm{cm}^{2}$ pump beam intersects the probe between 0.4 and $1.4 \mathrm{~ns}$ causing the probe to be amplified by a factor of 1.7 above the 'pump off' case.

Figure 5) A series of experiments measured the amplification of the probe beam as a function of the wavelength separation of the two beams as shown. The amplification is greatest when the frequency separation is in the vicinity of the prediction for the unshifted ion wave resonance, $\Delta \lambda=0.45 \pm 0.04 \mathrm{~nm}$.

Figure 6) The measured transmitted power is shown to be proportional to the probe intensity over a 5:1 range of $I_{\text {probe }} / I_{\text {pump }}$ using data obtained at disparate separation frequencies by normalizing to the amplification measured with $\mathrm{I}_{\text {probe }} / \mathrm{I}_{\text {pump }}=$ 0.13 .

Figure 7) Scale-1 hohlraum target with an $\mathrm{Au} / \mathrm{Be}$ patch on a portion of the wall, and two laser entrance holes (LEH) allowing illumination of the inside wall by ten Nova beams.

Figure 8) Time and spectral history of the scattered light from a laser beam incident on the $\mathrm{Au} / \mathrm{Be}$ patch in a scale-1 hohlraum filled with 1 atm. of $\mathrm{C}_{3} \mathrm{H}_{8}$. 
Figure 9) Time and spectrally integrated SBS and SRS reflectivities measured with $\mathrm{CH}_{4}$ filled hohlraum targets with varying percents of Be in the Au wall and varying types of beam smoothing. The SRS is observed to be more strongly dependent on the fraction of $B e(\sim$ ion wave damping rate) than the SBS

Figure 10) Measurements under the same conditions as Figure 10 but with a $\mathrm{C}_{3} \mathrm{H}_{8}$ gas fill show similar dependences on the fraction of the impurity.

Figure 11) Calculated electron density, temperature, and averaged ionization state, at $\mathrm{t}=0.7 \mathrm{~ns}$ for a gas bag target containing $90 \%$ $\mathrm{Xe}$ and $10 \% \mathrm{C}_{5} \mathrm{H}_{12}$ indicate the density and temperature scale lengths are long.

Figure 12) Time integrated reflectivity from an Xe filled 'gas bag' target with $5.5 \%$ impurity concentration shows a narrow peak at 575 $\mathrm{nm}$ in the 0.5 to $1.0 \mathrm{~ns}$ 'early' period.

Figure 13) SRS reflectivities from Xe filled 'gas bag' targets averaged over the $0.5 \mathrm{~ns}$ to $1.0 \mathrm{~ns}$ 'early' period and the $1.0 \mathrm{~ns}$ to $1.5 \mathrm{~ns}$ 'late' period are shown vs. impurity concentration. The impurity concentration is interpreted as the ion wave damping rate and the reflectivity is interpreted as the square of the fluctuation amplitude. Solid and dashed lines represent the EDI and LDI threshold amplitudes for a uniform beam and parameters 
relevant to early time data. The LDI for a non-uniform beam can be much lower as discussed in the text. 


\section{a) Stimulated Brillouin Scattering}

\section{(SBS)}

back scattered

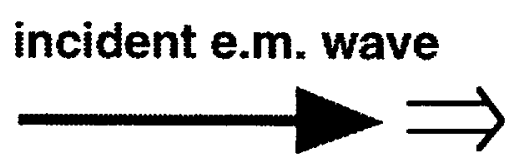

e. m. wave

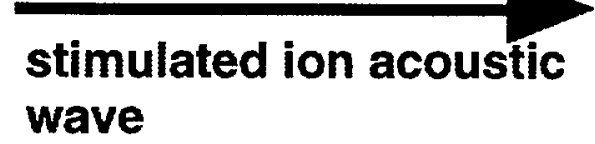

b) Stimulation by Crossing LaserBeams

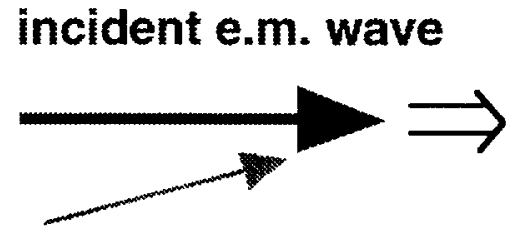

seeding e. m. wave

$$
\begin{aligned}
& \text { amplified } \\
& \text { seed wave }
\end{aligned}
$$

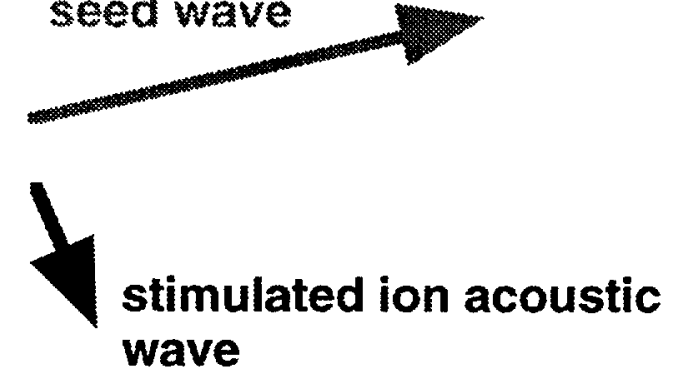

c) Stimulation by SRS generated Langmuir waves

incident e.m. wave

back scattered

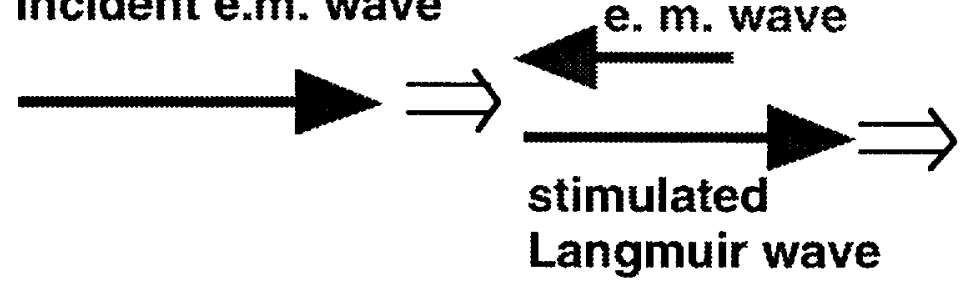

stimulated ion wave

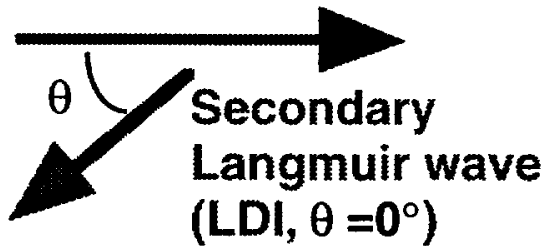
or e. m. wave (EDI, $\theta=90^{\circ}$ )

Figure 1 


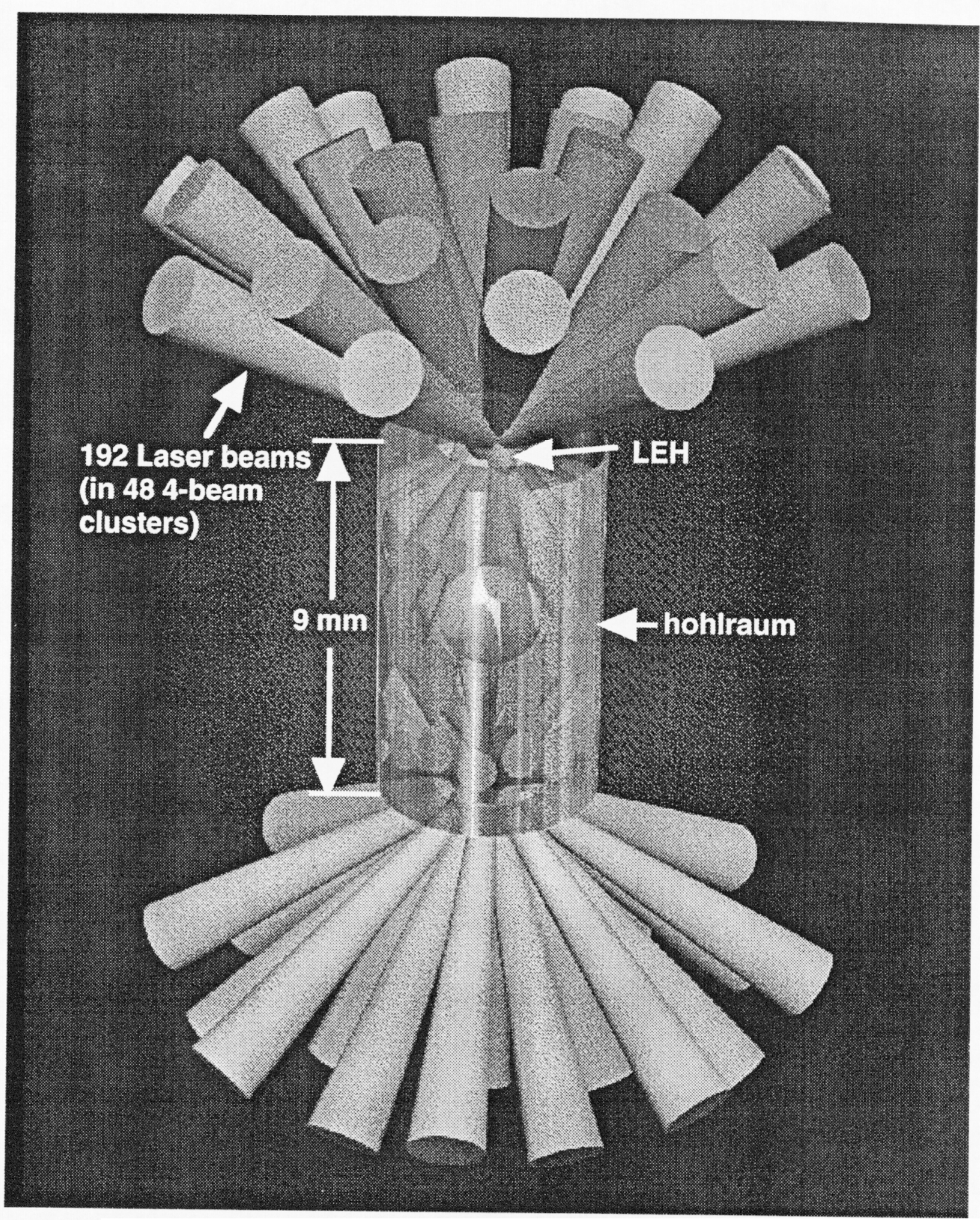

Figure 2a 


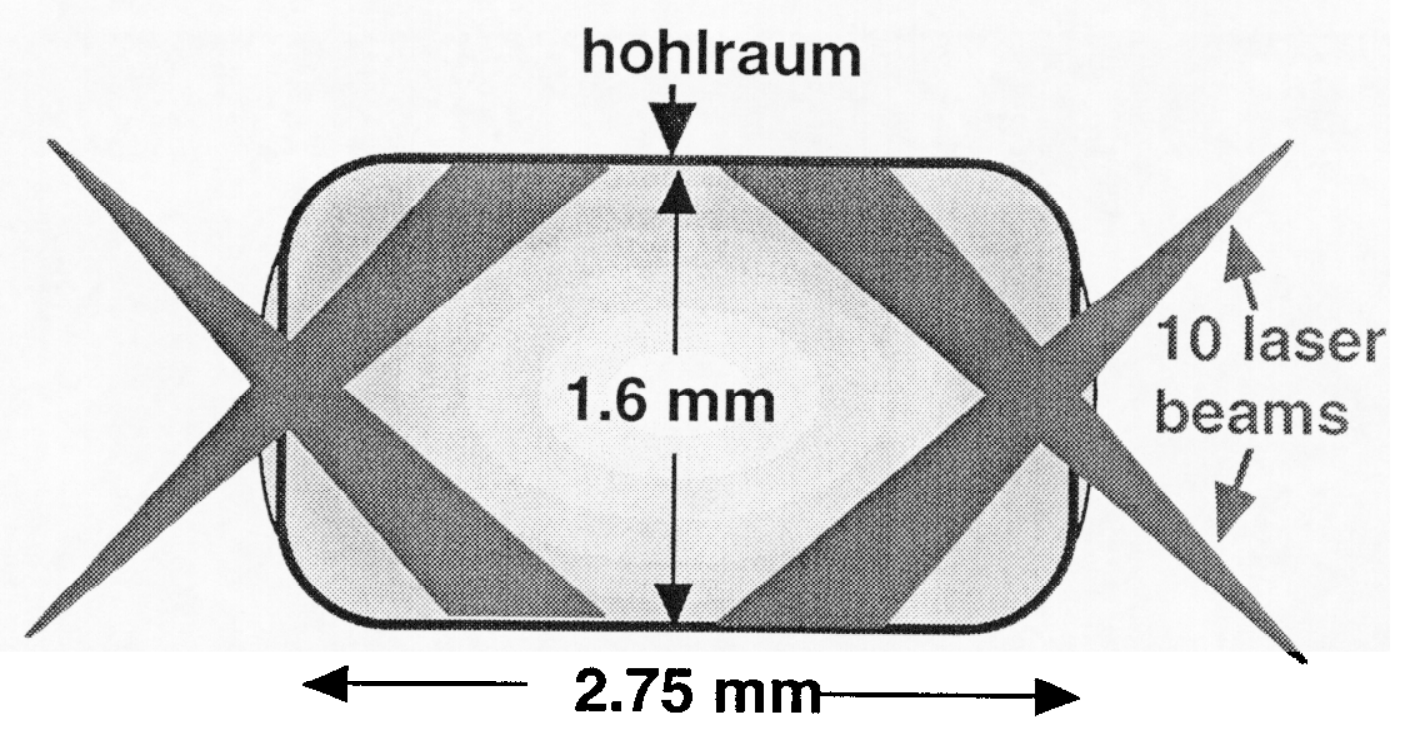

Figure 2b 


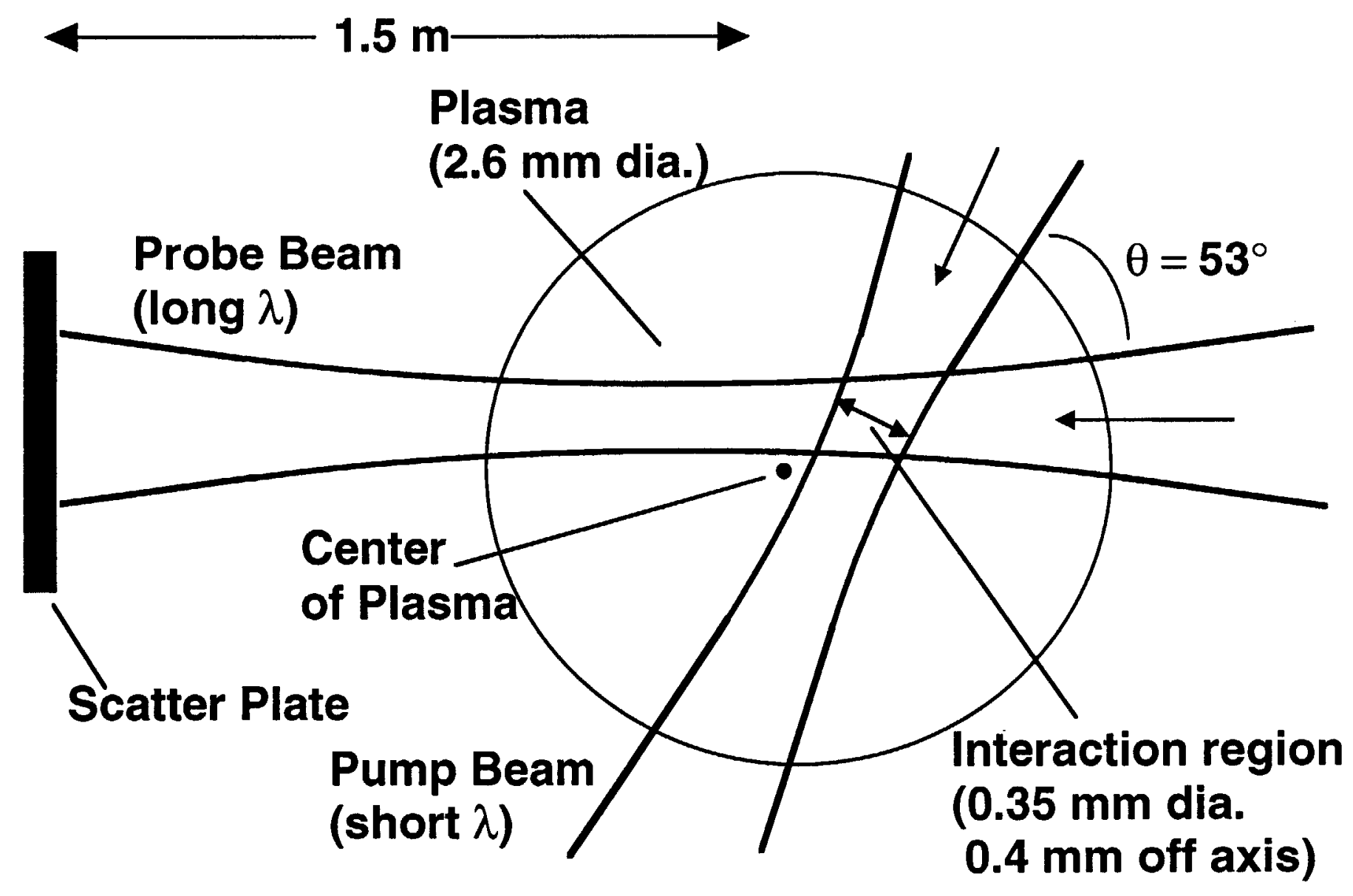

Figure 3 


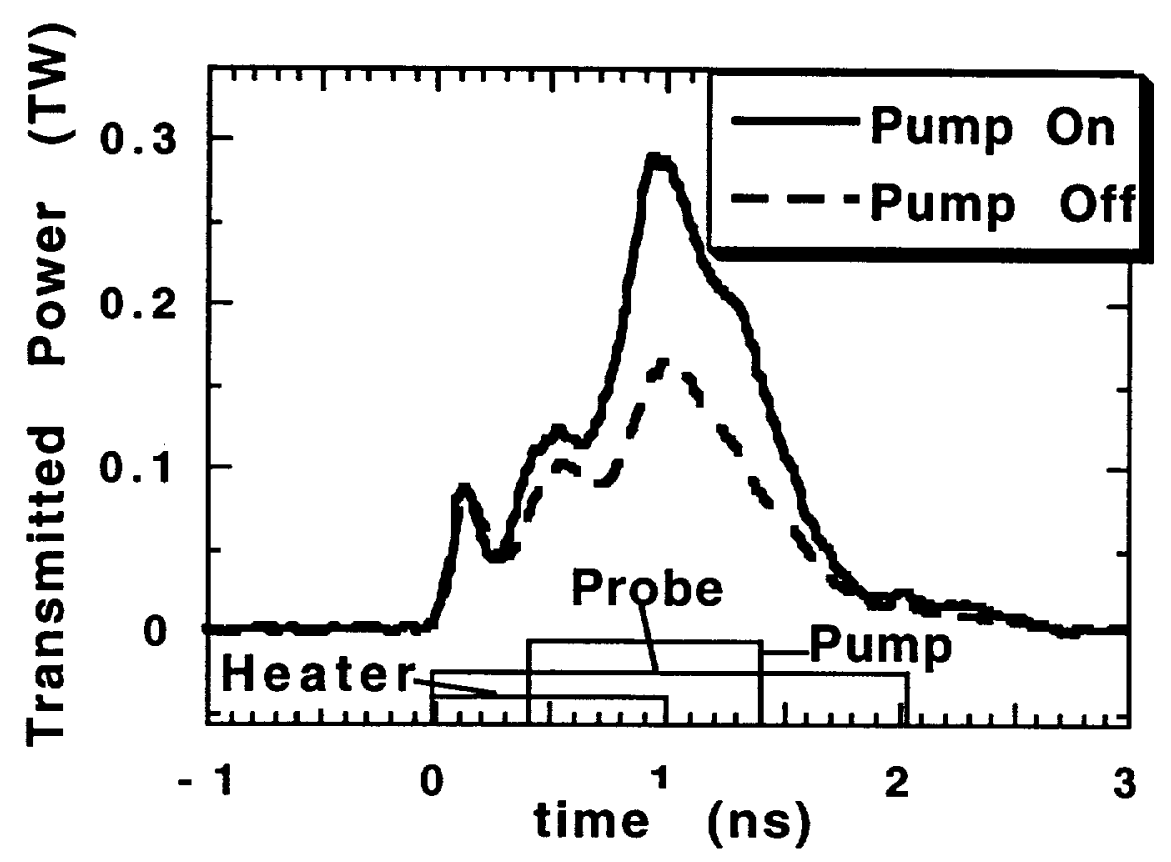

Figure 4 


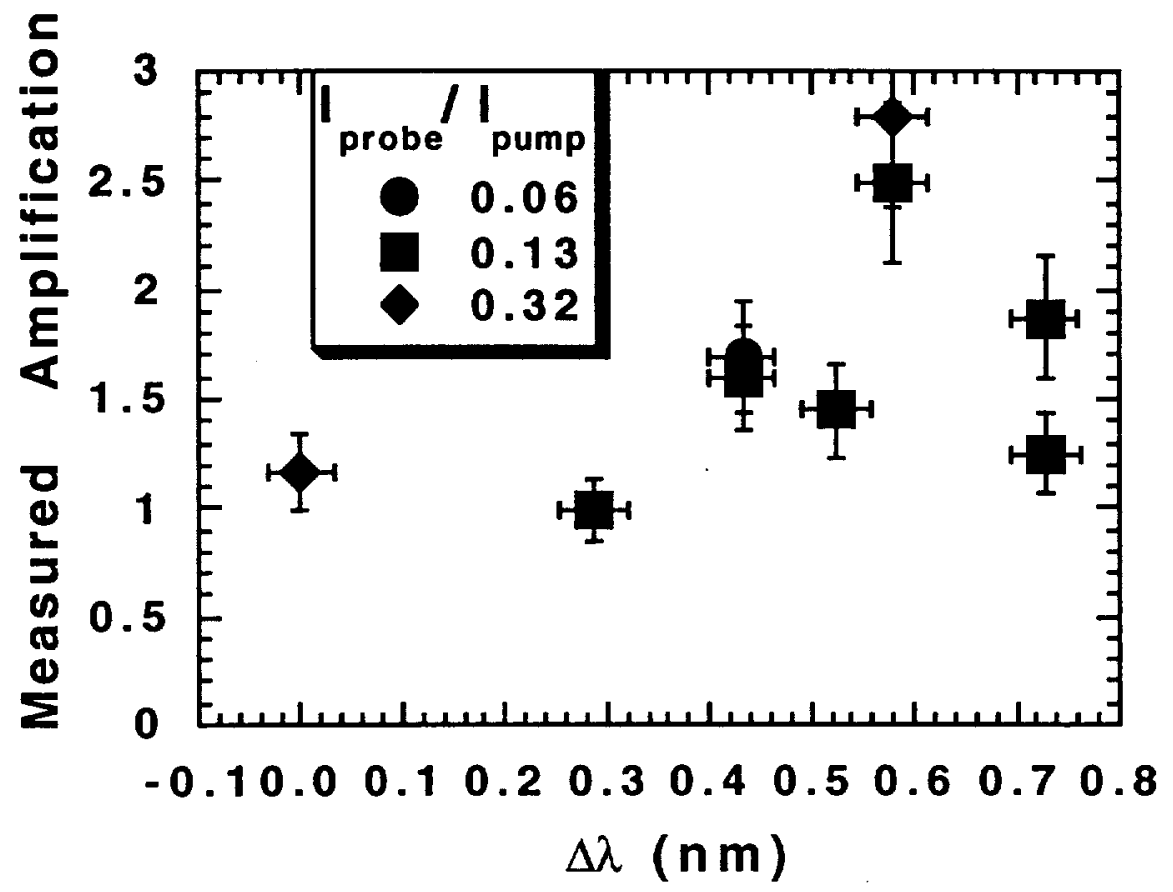

Figure 5 


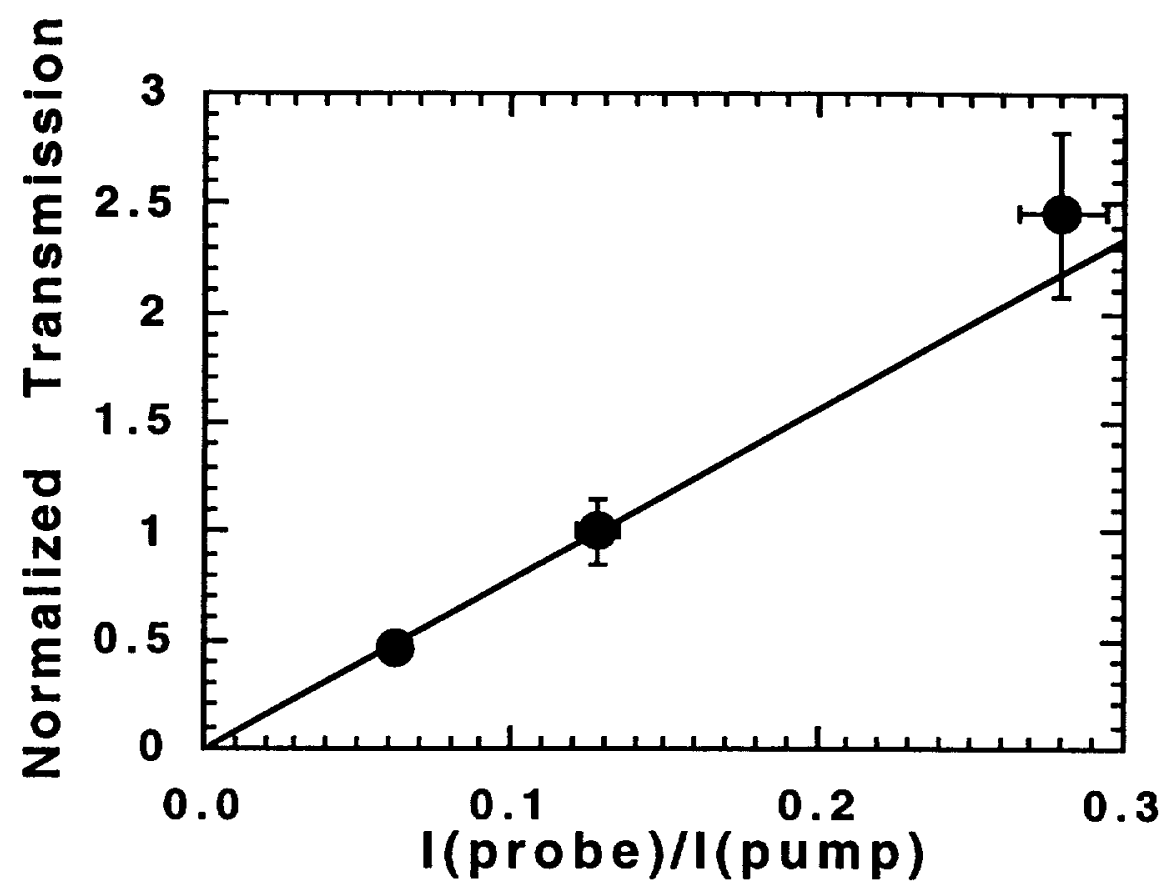

Figure 6 

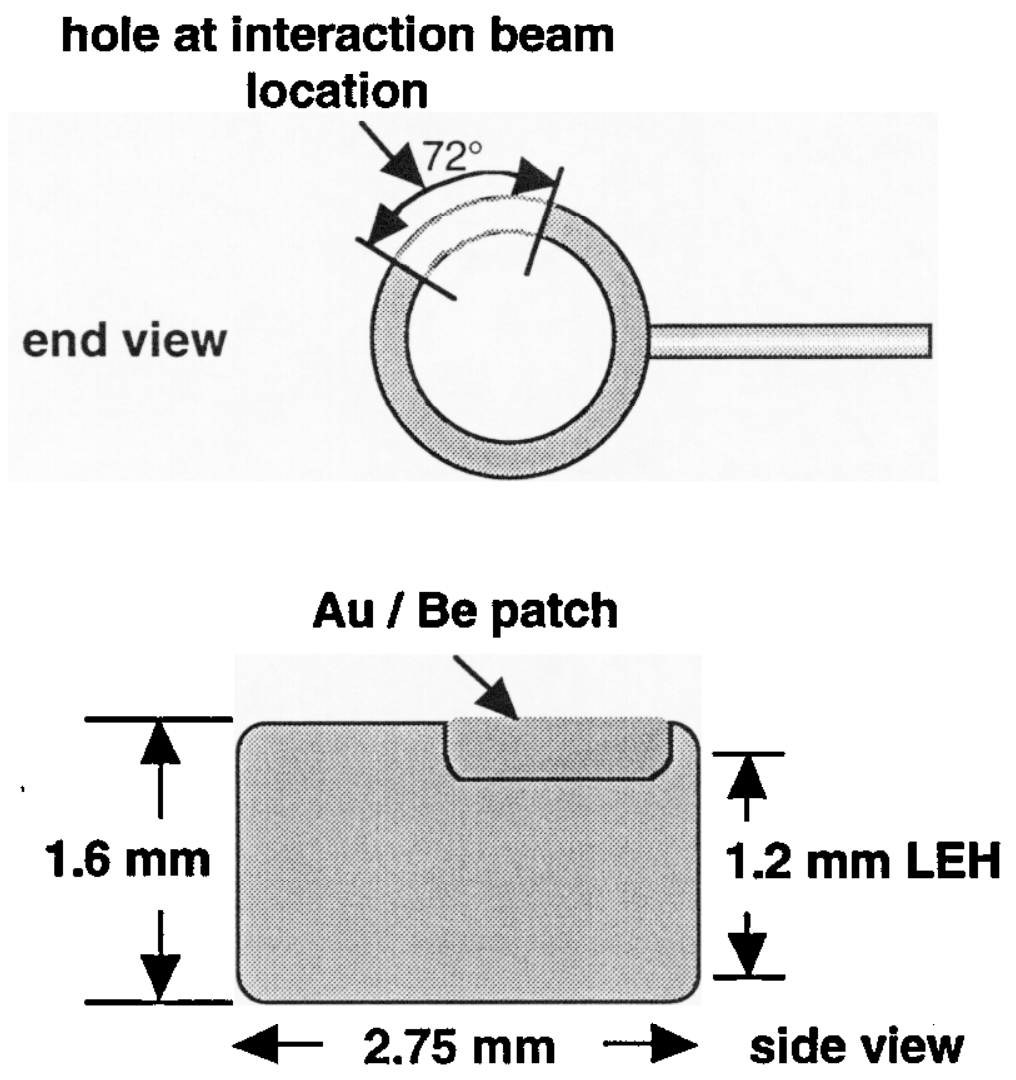

Figure 7 

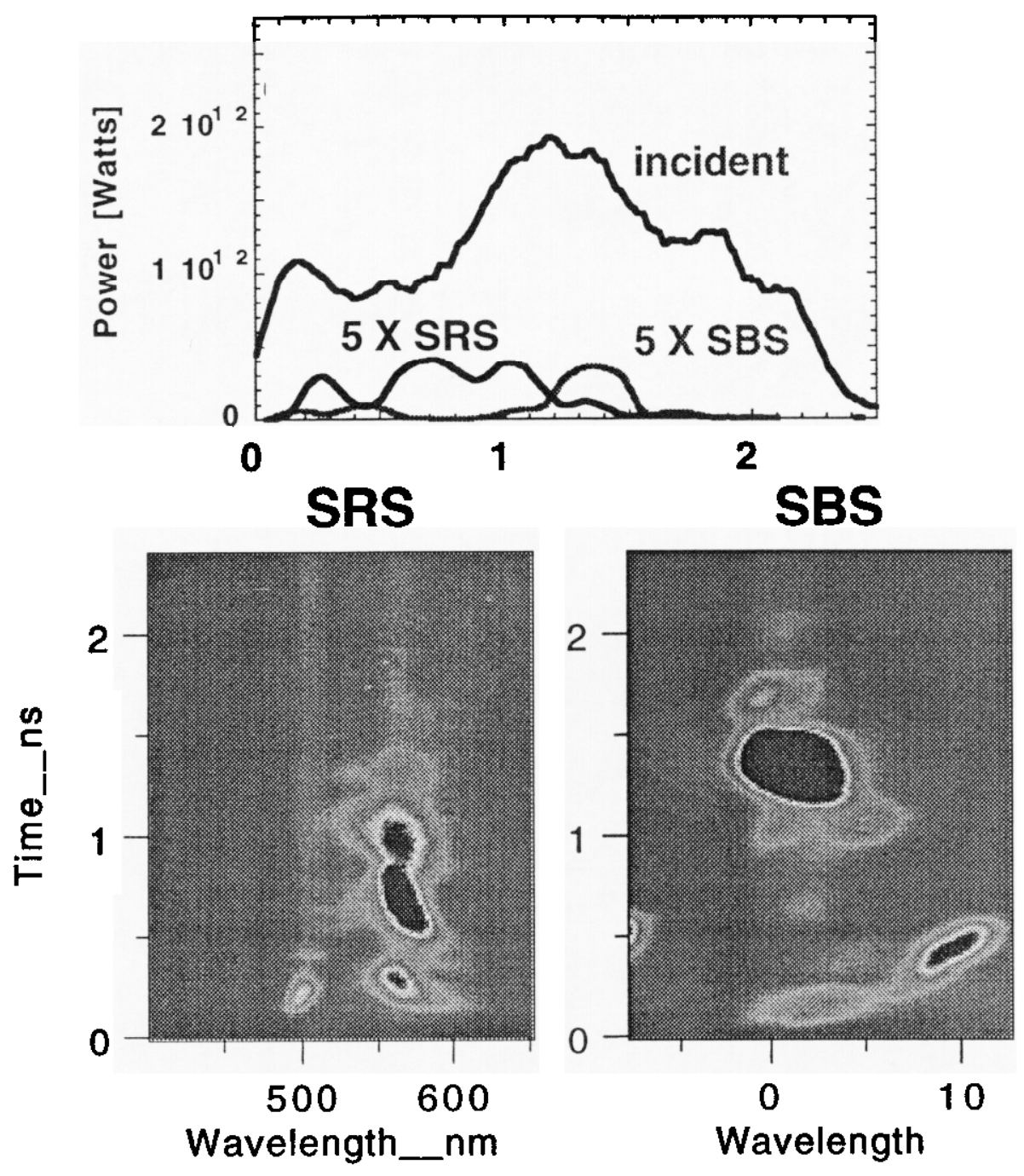

Figure 8 

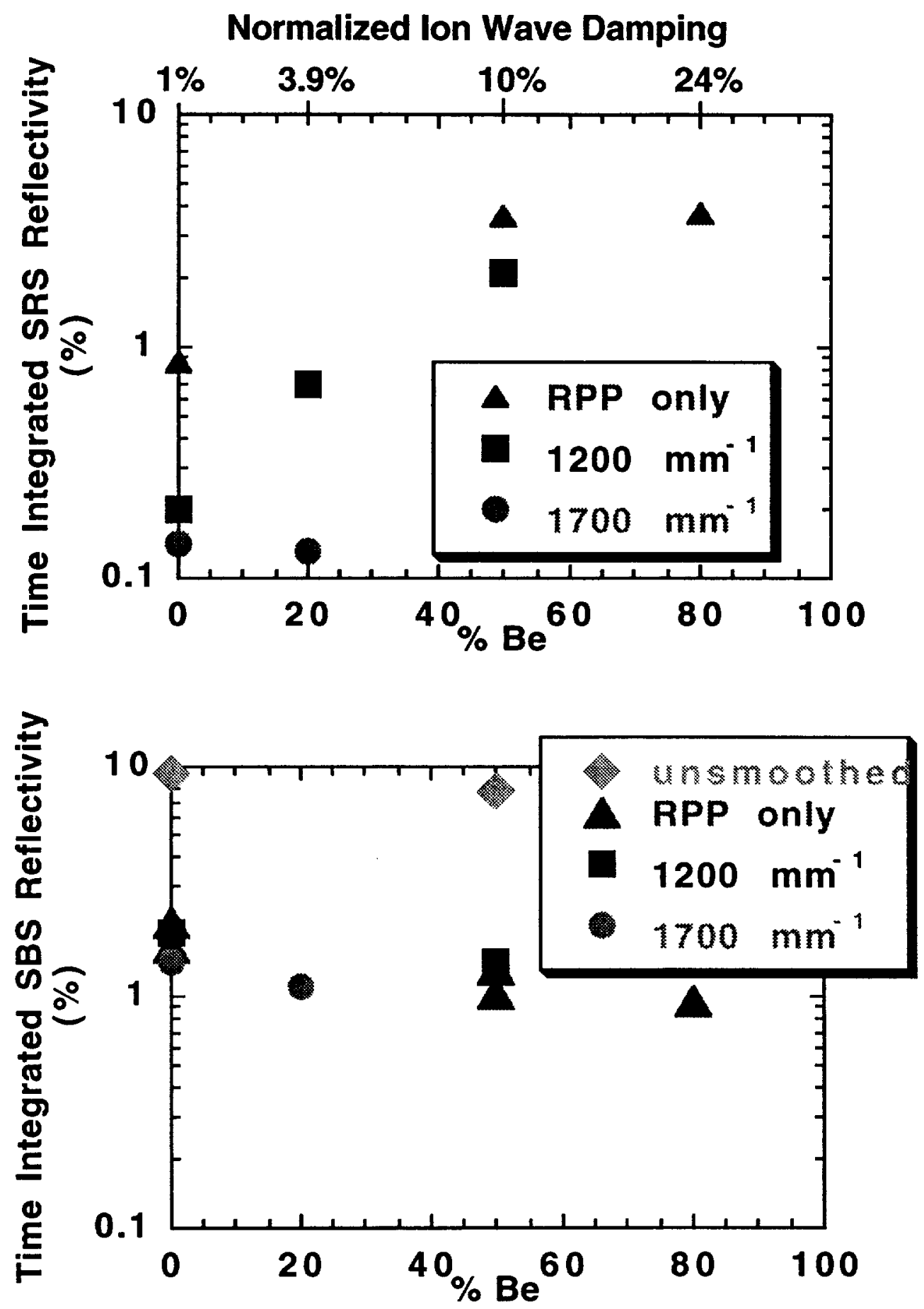

Figure 9 


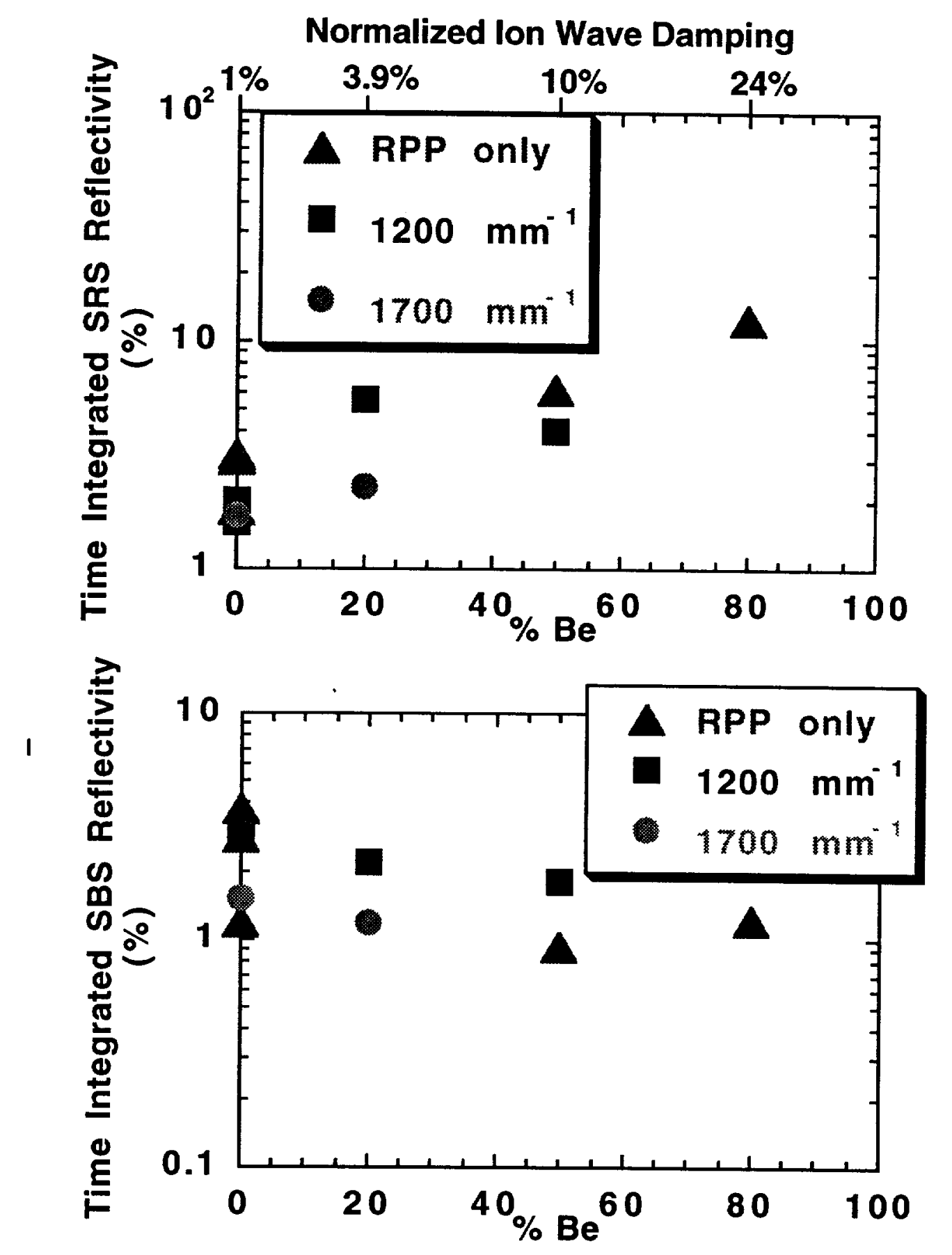

Figure 10 


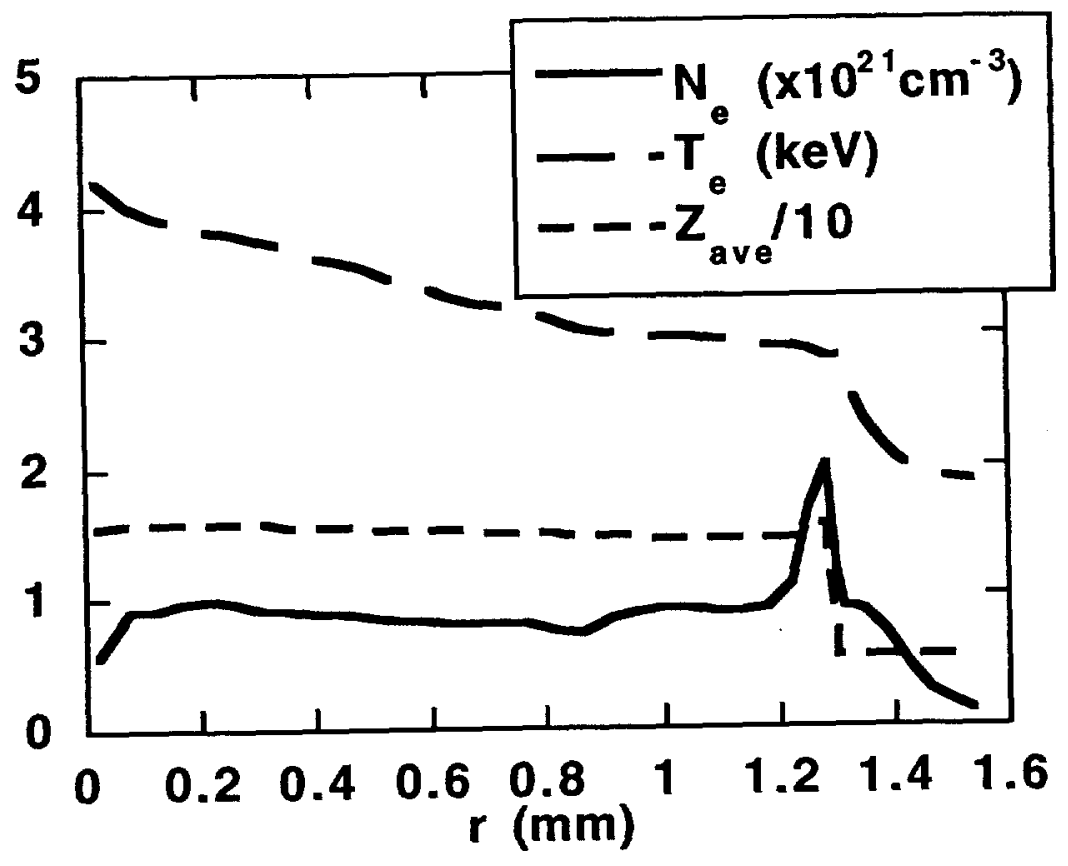

Figure 11 


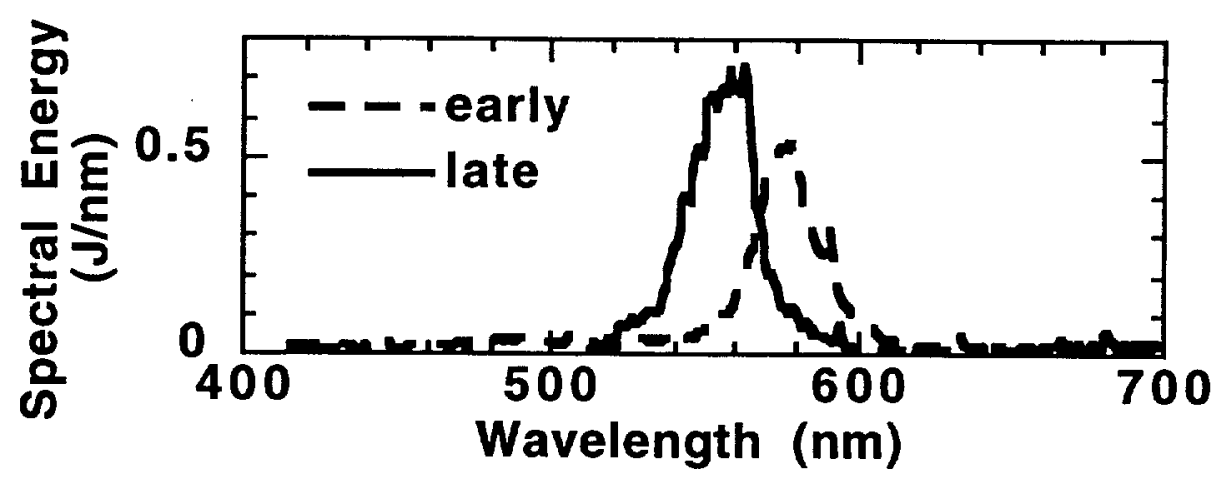

Figure 12

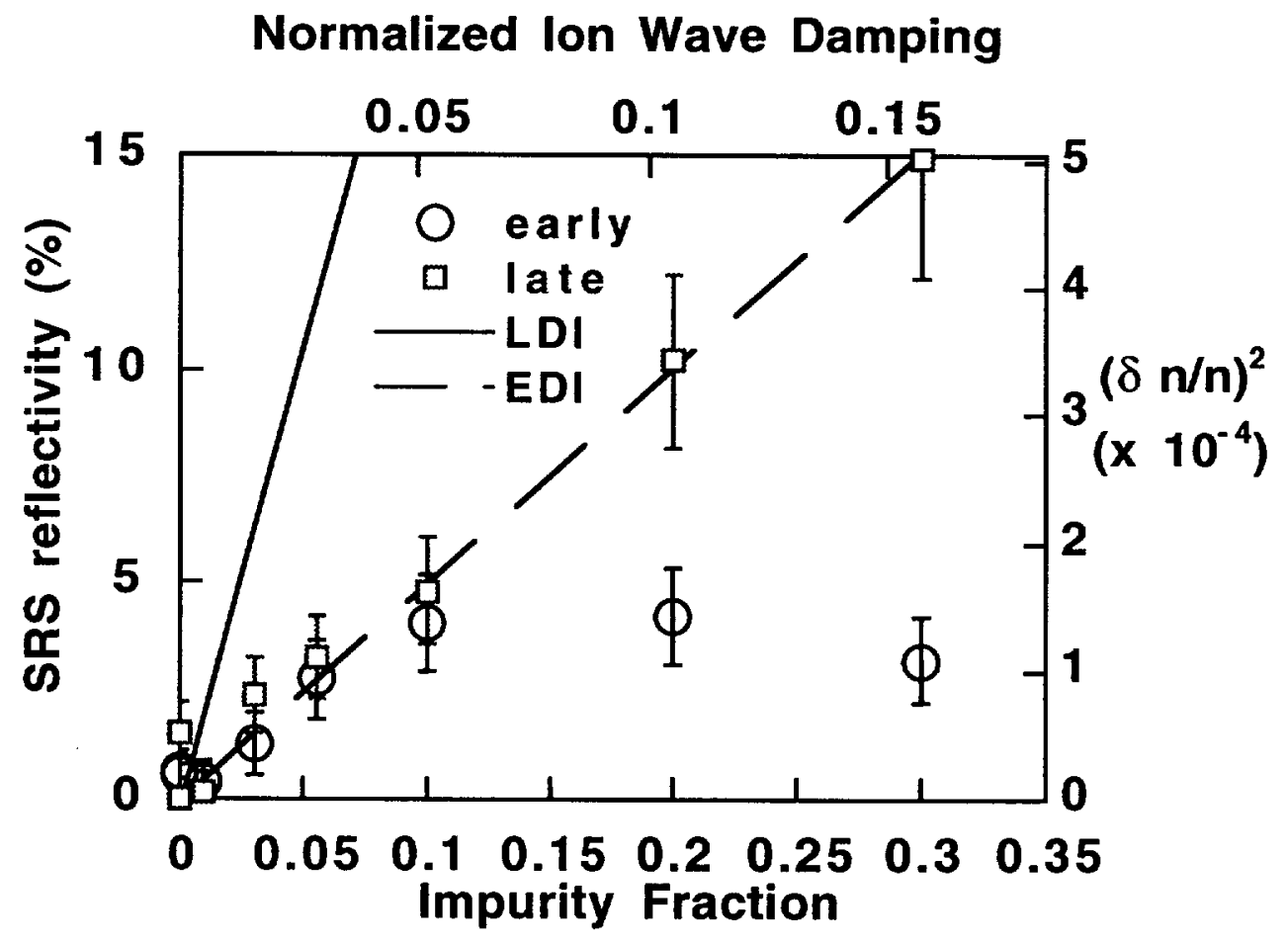

Figure 13 


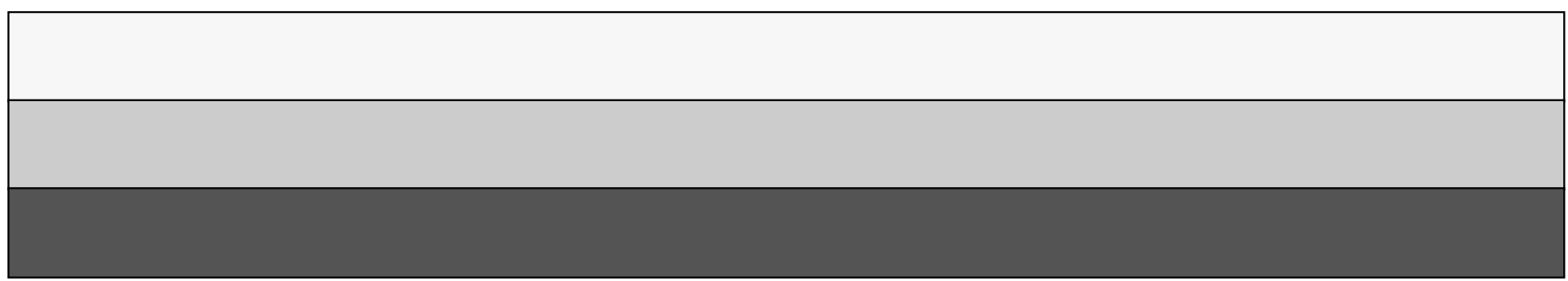

